\title{
Checklist das aves do Estado do Mato Grosso do Sul, Brasil
}

\author{
Alessandro Pacheco Nunes ${ }^{1}$, Fernando Costa Straube ${ }^{2,3}$, \\ Rudi Ricardo Laps ${ }^{3,4}$ \& Sérgio Roberto Posso ${ }^{3,4}$
}

\author{
1. Programa de Pós-graduação em Ecologia e Conservação/Universidade Federal do Mato Grosso do Sul, Campo Grande, MS, Brasil. (tiriba.ms@gmail.com) \\ 2. Hori Consultoria Ambiental, Curitiba, PR, Brasil. \\ 3. Comitê Brasileiro de Registros Ornitológicos (CBRO), Brasil. \\ 4. Universidade Federal de Mato Grosso do Sul, Três Lagoas, MS, Brasil.
}

Recebido 21 novembro 2016

Aceito 6 fevereiro 2017

DOI: $10.1590 / 1678-4766 e 2017154$

\begin{abstract}
Checklist of the birds of the state of Mato Grosso do Sul, Brazil. Contrasting with several other Brazilian states, just recently the state of Mato Grosso do Sul started to organize an inventory of its birdlife. We list 630 species of birds for the Mato Grosso do Sul, belonging to 26 orders and 74 families, representing $34 \%$ of birds occurring in Brazil. About $90 \%$ of these species have documented records of its occurrence in the state (Main List), according to proposed by CBRO. The others species still wait for adequate supporting documentation (Secondary List). Forty five species were excluded for the Main List and included in Tertiary List due to problems in supporting documentation and/or incompatible distribution. The variety of landscapes and vegetation types under the influence of the biomes Cerrado, Pantanal, Atlantic forest, Chaco and Bosque Chiquitano is partly responsible for the high species diversity in Mato Grosso do Sul. However, several of these landscapes have been suppressed and/or severely altered by pastures, agriculture (mostly monocultures of sugar cane and soybeans) and agroforestry (Pinus and Eucalyptus). Thirty-eight species of birds occurring in the state were present on red lists at the global and/or national level. There are still large gaps in knowledge about the avifauna of Mato Grosso do Sul, mainly in the region of Paiaguás in the Pantanal wetlands and in neighboring regions with the state of Goiás, Paraguay (Chaco) and Bolivia (Bosque Chiquitano). Institutions such as Embrapa Pantanal and the Universidade Federal do Mato Grosso do Sul, as well as the research of environmental consultants and birdwatchers, were relevant in the advancement of ornithological knowledge in the state. Much of the institutional museums harbor specimens collected in Mato Grosso do Sul, which highlights are the Museum of Comparative Zoology (Cambridge, USA), the American Museum of Natural History (New York), the Field Museum of Natural History (Chicago) and the National Museum of Natural History (Washington). In Brazil the main ornithological collections that keep material from Mato Grosso do Sul are the Museu de Zoologia (USP, São Paulo), the Museu Nacional (Rio de Janeiro) and the collection of Departmento de Zoologia at Universidade Federal de Minas Gerais (DZUFMG, Minas Gerais).
\end{abstract}

KEYWORDS. Pantanal, Cerrado, conservation status, biodiversity, Biota-MS Program.

RESUMO. Em contraste com vários outros estados brasileiros, apenas recentemente o Mato Grosso do Sul iniciou o inventário organizado de sua avifauna. Relacionamos 630 espécies de aves para o Mato Grosso do Sul, pertencentes a 26 ordens e 74 famílias, que corresponde a 34\% da avifauna ocorrente no Brasil. Cerca de $90 \%$ dessas espécies apresentam registros comprobatórios de ocorrência no estado conforme as normas propostas pelo CBRO. A grande diversidade de fitofisionomias e paisagens sob influência dos biomas Cerrado, Pantanal, Chaco, Bosques Chiquitanos e Mata Atlântica são responsáveis em parte, pela diversidade de espécies ocorrentes no Mato Grosso do Sul. No entanto, várias dessas fitofisionomias e paisagens já foram suprimidas e/ou alteradas pela pecuária e principalmente monoculturas (Pinus, Eucalyptus e cana-de-açúcar). Trinta e oito espécies de aves ocorrentes no estado encontram-se presentes em listas de espécies ameaçadas de extinção em âmbito global e/ou nacional. Ainda existem grandes lacunas de conhecimento sobre a avifauna sul-matogrossense, notadamente no Pantanal do Paiaguás e em regiões limítrofes com o estado de Goiás e países como Paraguai (Chaco) e a Bolívia (Bosques Chiquitanos). Instituiç̃es como Embrapa Pantanal e Universidade Federal de Mato Grosso do Sul, bem como o trabalho de consultores ambientais e observadores de aves foram relevantes no avanço do conhecimento sobre a avifauna no estado. Grande parte dos museus institucionais no exterior abriga exemplares coletados no Mato Grosso do Sul, dentre os quais se destacam o Museum of Comparative Zoology (Cambridge, EUA), o American Museum of Natural History (Nova York, EUA), o Field Museum of Natural History (Chicago, EUA) e o National Museum of Natural History (Washington, DC, EUA). No Brasil as principais coleções que guardam material ornitológico sul-matogrossense são o Museu de Zoologia (USP, São Paulo), o Museu Nacional (UFRJ, Rio de Janeiro) e o Departamento de Zoologia da Universidade Federal de Minas Gerais (DZUFMG, Minas Gerais).

PALAVRAS-CHAVE. Pantanal, Cerrado, biodiversidade, Programa BIOTA-MS.

Listas faunísticas são muito importantes para o avanço das ciências naturais, como fontes indispensáveis de subsídios a outras áreas do conhecimento, como a biogeografia, a ecologia e mesmo seus desdobramentos, como a biologia da conservação e as avaliações de impacto ambiental (SILVEIRA et al., 2010; SCHERER-NeTo et al., 2011). De fato, todo o conhecimento sobre a distribuição das aves brasileiras começou a ser estabelecido, ao longo da história, com o acúmulo de dados originários dos tantos estudos de natureza regional (PACHECO \& BAUER, 2001).

Por várias razões, o Mato Grosso do Sul apenas recentemente iniciou o inventário organizado de sua avifauna, contrastando fortemente com outros estados brasileiros como São Paulo (IHERING, 1898, 1900, 1904), Rio Grande do Sul 
(Ihering, 1899), Minas Gerais (Pinto, 1952) e Espírito Santo (RuschI, 1953). Este estudo, fundamentado como fonte de referência, objetiva relacionar as espécies de aves até o momento registrados no Estado de Mato Grosso do Sul.

\section{MATERIAL E MÉTODOS}

Para a elaboração deste checklist, que deve ser tratado como uma ferramenta preliminar e suscetível a alterações, foram considerados registros visuais, audiovisuais e/ou sonoros disponíveis na literatura, museus institucionais no Brasil e no exterior, bem como os dados obtidos em campo pelos autores em diversas localidades do estado.

Ela foi construída com base no formato da "Lista das Aves do Brasill", compilação que é anualmente produzida e atualizada pelo Comitê Brasileiro de Registros Ornitológicos (CBRO, 2011), grupo de estudos da Sociedade Brasileira de Ornitologia. Sob essa chancela, o procedimento aqui adotado inclui tanto a apresentação quanto os critérios adotados por aquele Comitê, com dois limites bem claros e definidos para a admissão de espécies ao inventário: (1) Lista Primária (LP), que agrega espécies com pelo menos um dos registros de ocorrência no Mato Grosso do Sul provido de evidência documental, ou seja, item(ns) disponível(is) para consulta independente, na forma de espécime integral ou parcial, fotografia, gravação de áudio ou vídeo, que permitam a determinação segura e indiscutível do táxon e (2) Lista Secundária (LS), incluindo espécies contando com um ou mais registros para o território sul-mato-grossense, mas cuja evidência documental não é conhecida ou disponível. Neste contexto, o protocolo de filtragem é o detalhamento da informação alusiva ao contato, seja visual ou auditivo, associado obrigatoriamente à coerência com o padrão distribucional e de dispersão da espécie estabelecido com base em evidências documentais. As espécies incluídas à lista estadual sob esse critério encontram-se discriminadas entre colchetes.

Em virtude do objetivo deste estudo, não se encontram indicados elementos fundamentais para o juízo e conclusões aqui procedidos, informações essas que estão em vias de compilação em obra especialmente destinada e esse fim (NuNES, et al., 2007).

Adicionalmente, e também conforme recomendações do CBRO, são excluídas algumas espécies que, embora citadas na literatura como componentes da avifauna do Mato Grosso do Sul, carecem de detalhes documentados ou minimamente circunstanciados. Esses casos estão consolidados em uma Lista Terciária (Apêndice 1), que também aponta a fonte original de menção e a racionália respectiva para a exclusão.

A lista de espécies segue a ordenação taxonômica bem como a nomenclatura científica e os nomes em português propostos pelo Comitê Brasileiro de Registros Ornitológicos (CBRO, 2011), com exceção de algumas recentes deliberações para as famílias Caprimulgidae e Thraupidae/Emberizidae e, ainda, de situações provisórias (incertae sedis) de alguns grupos ou espécies, nesses casos baseados na edição anterior da mesma lista (CBRO, 2009), sob a forma de arranjo mais coerente e consensual possível.

Adicionalmente, este estudo adota os limites de espécies para Aburria Reichenbach, 1853 apresentados por LOPES (2009) contra GRAU et al. (2004) e CBRO (2011), particularmente na aceitação de $A$. grayi Pelzeln, 1870 e A. nattereri Reichenbach, 1862 como espécies plenas. Além disso, também se utiliza os conceitos e conclusões de D’Horta et al. (2008) na admissão de quatro espécies antes agregadas em Icterus cayanensis (Linnaeus, 1766), tais como I. pyrrhopterus (Vieillot, 1819), I. valenciobuenoi Ihering, 1902; I. periporphyrus (Bonaparte, 1850) e I. tibialis Swainson, 1838, sendo as três primeiras ocorrentes no Mato Grosso do Sul (vide OmLand et al., 1999; Sturge et al., 2009). Essas manobras, embora discordantes do cenário nacional, visam ao fortalecimento do conhecimento da distribuição dos respectivos táxons envolvidos, todos eles - na opinião dos autores - perfeitamente reconhecíveis e dispondo de documentações satisfatórias.

Por questões de objetividade, os nomes em português aludem exclusivamente aos "nomes vernáculos técnicos" (sensu Farias \& Alves, 2007). Cientes de que se tratam de denominações técnicas escolhidas para individualizar cada espécie de ave brasileira com uma única designação em língua portuguesa, acreditamos que tais nomes possuem caráter puramente instrumental, visando tão somente à comunicação com o público leigo. Nomes populares autênticos ("nomes locais", segundo FARIAS \& ALVES, 2007) desta forma, não são apresentados, aguardando estudos pertinentes de colecionamento geograficamente significativo e a necessária base linguística, visando especialmente à preservação desse patrimônio cultural de indiscutível importância.

Na elaboração da lista de espécies de aves ameaçadas de extinção ocorrentes no Mato Grosso do Sul, consideramos a lista nacional de espécies ameaçadas (SilveIra \& Straube, 2008), com os seguintes status de ameaça: CR (Criticamente em Perigo), EN (Em Perigo) e VU (Vulnerável).

\section{RESULTADOS E DISCUSSÃO}

Relacionamos 630 espécies de aves para o Mato Grosso do Sul, pertencentes a 26 ordens, 74 famílias e 396 gêneros (Tab. I), o que corresponde a aproximadamente 35\% das espécies ocorrentes no Brasil (CBRO, 2011).

Aproximadamente $90 \%$ das espécies de aves ocorrentes no estado apresentam registros comprobatórios de ocorrência por meio de espécime(s) integral(is) ou parcial(is), fotografia, gravação de áudio ou vídeo e outros tipos de documentos que permitam a determinação segura, e a aferição posterior, do táxon (vide CARLOS et al., 2010). As demais ainda aguardam documentação adequada, mas foram incluídas provisoriamente na lista secundária. Quarenta e seis espécies foram transferidas para a lista terciária devido à ausência (ou casos insolúveis mal esclarecidos) de documentação, associada à incompatibilidade de distribuição geográfica (Apêndice 1). 
Tab. I. Lista das aves do estado do Mato Grosso do Sul (Brasil). Status de conservação: CR (Criticamente em Perigo), EN (Em Perigo) e VU (Vulnerável), segundo Silveira \& Straube (2008).

\begin{tabular}{|c|c|}
\hline Nome do táxon & Nome popular \\
\hline \multirow{2}{*}{\multicolumn{2}{|c|}{$\begin{array}{l}\text { STRUTHIONIFORMES } \\
\text { Rheidae }\end{array}$}} \\
\hline & \\
\hline Rhea americana (Linnaeus, 1758) & ema \\
\hline \multicolumn{2}{|l|}{ TINAMIFORMES } \\
\hline \multicolumn{2}{|l|}{ Tinamidae } \\
\hline Tinamus solitarius (Vieillot, 1819) & macuco \\
\hline [Crypturellus soui (Hermann, 1783)] & tururim \\
\hline Crypturellus obsoletus (Temminck, 1815) & inhambuguaçu \\
\hline Crypturellus undulatus (Temminck, 1815) & jaó \\
\hline Crypturellus parvirostris (Wagler, 1827) & inhambu-chororó \\
\hline Crypturellus tataupa (Temminck, 1815) & inhambu-chintã \\
\hline Rhynchotus rufescens (Temminck, 1815) & perdiz \\
\hline Nothura minor (Spix, 1825) vU & codorna-mineira \\
\hline Nothura maculosa (Temminck, 1815) & codorna-amarela \\
\hline$\left[\right.$ Taoniscus nanus $\left(\right.$ Temminck, 1815)] ${ }^{\mathrm{VU}}$ & inhambu-carapé \\
\hline \multicolumn{2}{|l|}{ ANSERIFORMES } \\
\hline \multicolumn{2}{|l|}{ Anhimidae } \\
\hline Anhima cornuta (Linnaeus, 1766) & anhuma \\
\hline Chauna torquata (Oken, 1816) & tachã \\
\hline \multicolumn{2}{|l|}{ Anatidae } \\
\hline Dendrocygna bicolor (Vieillot, 1816) & marreca-caneleira \\
\hline Dendrocygna viduata (Linnaeus, 1766) & irerê \\
\hline Dendrocygna autumnalis (Linnaeus, 1758) & asa-branca \\
\hline Coscoroba coscoroba (Molina, 1782) & capororoca \\
\hline Neochen jubata (Spix, 1825) & pato-corredor \\
\hline Cairina moschata (Linnaeus, 1758) & pato-do-mato \\
\hline Sarkidiornis sylvicola Ihering \& Ihering, 1907 & pato-de-crista \\
\hline Callonetta leucophrys (Vieillot, 1816) & marreca-de-coleira \\
\hline Amazonetta brasiliensis (Gmelin, 1789) & pé-vermelho \\
\hline Anas bahamensis Linnaeus, 1758 & marreca-toicinho \\
\hline [Anas versicolor Vieillot, 1816] & marreca-cricri \\
\hline Anas platalea Vieillot, 1816 & marreca-colhereira \\
\hline [Netta erythrophthalma (Wied, 1832)] & paturi-preta \\
\hline Netta peposaca (Vieillot, 1816) & marrecão \\
\hline Nomonyx dominica (Linnaeus, 1766) & marreca-de-bico-roxo \\
\hline Oxyura vittata (Philippi, 1860) & marreca-pé-na-bunda \\
\hline \multicolumn{2}{|l|}{ GALLIFORMES } \\
\hline \multicolumn{2}{|l|}{ Cracidae } \\
\hline Ortalis guttata (Spix, 1825) & aracuã \\
\hline Ortalis canicollis (Wagler, 1830) & aracuã-do-pantanal \\
\hline Penelope superciliaris Temminck, 1815 & jacupemba \\
\hline Penelope ochrogaster Pelzeln, 1870 vu & jacu-de-barriga-castanha \\
\hline Aburria grayi Pelzeln, 1870 & jacutinga-de-garganta-azul \\
\hline Aburria nattereri Reichenbach, 1862 & cujubi \\
\hline Crax fasciolata Spix, 1825 & mutum-de-penacho \\
\hline \multicolumn{2}{|l|}{ Odontophoridae } \\
\hline Odontophorus capueira (Spix, 1825) & uru \\
\hline \multicolumn{2}{|l|}{ PODICIPEDIFORMES } \\
\hline \multicolumn{2}{|l|}{ Podicipedidae } \\
\hline [Rollandia rolland (Quoy \& Gaimard, 1824)] & mergulhão-de-orelha-branca \\
\hline Tachybaptus dominicus (Linnaeus, 1766) & mergulhão-pequeno \\
\hline Podilymbus podiceps (Linnaeus, 1758) & mergulhão-caçador \\
\hline \multicolumn{2}{|l|}{ CICONIIFORMES } \\
\hline \multicolumn{2}{|l|}{ Ciconiidae } \\
\hline Ciconia maguari (Gmelin, 1789) & maguari \\
\hline Jabiru mycteria (Lichtenstein, 1819) & tuiuiú \\
\hline Mycteria americana Linnaeus, 1758 & cabeça-seca \\
\hline \multicolumn{2}{|l|}{ SULIFORMES } \\
\hline \multicolumn{2}{|l|}{ Phalacrocoracidae } \\
\hline Phalacrocorax brasilianus (Gmelin, 1789) & biguá \\
\hline Anhingidae & \\
\hline Anhinga anhinga (Linnaeus, 1766) & biguatinga \\
\hline PELECANIFORMES & \\
\hline Ardeidae & \\
\hline Tigrisoma lineatum (Boddaert, 1783) & socó-boi \\
\hline
\end{tabular}


Tab. I. Cont.

\begin{tabular}{|c|c|}
\hline Nome do táxon & Nome popular \\
\hline Tigrisoma fasciatum $\left(\right.$ Such, 1825) ${ }^{\mathrm{EN}}$ & socó-boi-escuro \\
\hline Agamia agami (Gmelin, 1789) & garça-da-mata \\
\hline Cochlearius cochlearius (Linnaeus, 1766) & arapapá \\
\hline [Botaurus pinnatus (Wagler, 1829)] & socó-boi-baio \\
\hline [Ixobrychus exilis (Gmelin, 1789)] & socoí-vermelho \\
\hline [Ixobrychus involucris (Vieillot, 1823)] & socoí-vermelho \\
\hline Nycticorax nycticorax (Linnaeus, 1758) & savacu \\
\hline Butorides striata (Linnaeus, 1758) & socozinho \\
\hline Bubulcus ibis (Linnaeus, 1758) & garça-vaqueira \\
\hline Ardea cocoi Linnaeus, 1766 & garça-moura \\
\hline Ardea alba Linnaeus, 1758 & garça-branca-grande \\
\hline Syrigma sibilatrix (Temminck, 1824) & maria-faceira \\
\hline Pilherodius pileatus (Boddaert, 1783) & garça-real \\
\hline Egretta thula (Molina, 1782) & garça-branca-pequena \\
\hline Egretta caerulea (Linnaeus, 1758) & garça-azul \\
\hline \multicolumn{2}{|l|}{ Threskiornithidae } \\
\hline Plegadis chihi (Vieillot, 1817) & caraúna-de-cara-branca \\
\hline Mesembrinibis cayennensis (Gmelin, 1789) & coró-coró \\
\hline Phimosus infuscatus (Lichtenstein, 1823) & tapicuru-de-cara-pelada \\
\hline Theristicus caerulescens (Vieillot, 1817) & maçarico-real \\
\hline Theristicus caudatus (Boddaert, 1783) & curicaca \\
\hline Platalea ajaja Linnaeus, 1758 & colhereiro \\
\hline \multicolumn{2}{|l|}{ CATHARTIFORMES } \\
\hline \multicolumn{2}{|l|}{ Cathartidae } \\
\hline Cathartes aura (Linnaeus, 1758) & urubu-de-cabeça-vermelha \\
\hline Cathartes burrovianus Cassin, 1845 & urubu-de-cabeça-amarela \\
\hline Coragyps atratus (Bechstein, 1793) & urubu-de-cabeça-preta \\
\hline Sarcoramphus papa (Linnaeus, 1758) & urubu-rei \\
\hline \multicolumn{2}{|l|}{ ACCIPITRIFORMES } \\
\hline \multicolumn{2}{|l|}{ Pandionidae } \\
\hline Pandion haliaetus (Linnaeus, 1758) & águia-pescadora \\
\hline \multicolumn{2}{|l|}{ Accipitridae } \\
\hline Leptodon cayanensis (Latham, 1790) & gavião-de-cabeça-cinza \\
\hline Chondrohierax uncinatus (Temminck, 1822) & caracoleiro \\
\hline Elanoides forficatus (Linnaeus, 1758) & gavião-tesoura \\
\hline Gampsonyx swainsonii Vigors, 1825 & gaviãozinho \\
\hline Elanus leucurus (Vieillot, 1818) & gavião-peneira \\
\hline Harpagus diodon (Temminck, 1823) & gavião-bombachinha \\
\hline Harpagus bidentatus (Latham, 1790) & gavião-ripina \\
\hline Circus buffoni (Gmelin, 1788) & gavião-do-banhado \\
\hline Accipiter poliogaster (Temminck, 1824) & tauató-pintado \\
\hline [Accipiter superciliosus (Linnaeus, 1766)] & gavião-miudinho \\
\hline Accipiter striatus Vieillot, 1808 & gavião-miúdo \\
\hline Accipiter bicolor (Vieillot, 1817) & gavião-bombachinha-grande \\
\hline Ictinia mississippiensis (Wilson, 1811) & sauveiro-do-norte \\
\hline Ictinia plumbea (Gmelin, 1788) & sovi \\
\hline Busarellus nigricollis (Latham, 1790) & gavião-belo \\
\hline Rostrhamus sociabilis (Vieillot, 1817) & gavião-caramujeiro \\
\hline Geranospiza caerulescens (Vieillot, 1817) & gavião-pernilongo \\
\hline Heterospizias meridionalis (Latham, 1790) & gavião-caboclo \\
\hline Urubitinga urubitinga (Gmelin, 1788) & gavião-preto \\
\hline Urubitinga coronata (Vieillot, 1817) & águia-cinzenta \\
\hline Rupornis magnirostris (Gmelin, 1788) & gavião-carijó \\
\hline Parabuteo unicinctus (Temminck, 1824) & gavião-asa-de-telha \\
\hline Geranoaetus albicaudatus Vieillot, 1816 & gavião-de-rabo-branco \\
\hline Geranoaetus melanoleucus (Vieillot, 1819) & águia-chilena \\
\hline Pseudastur albicollis (Latham, 1790) & gavião-branco \\
\hline Buteo nitidus (Latham, 1790) & gavião-pedrês \\
\hline Buteo platypterus (Vieillot, 1823) & gavião-de-asa-larga \\
\hline Buteo brachyurus Vieillot, 1816 & gavião-de-cauda-curta \\
\hline Buteo albonotatus Kaup, 1847 & gavião-de-rabo-barrado \\
\hline [Morphnus guianensis (Daudin, 1800)] & uiraçu-falso \\
\hline Harpia harpyja (Linnaeus, 1758) & gavião-real \\
\hline Spizaetus tyrannus (Wied, 1820) & gavião-pega-macaco \\
\hline Spizaetus melanoleucus (Vieillot, 1816) & gavião-pato \\
\hline Spizaetus ornatus (Daudin, 1800) & gavião-de-penacho \\
\hline
\end{tabular}


Tab. I. Cont.

\begin{tabular}{|c|c|}
\hline \multirow{2}{*}{\multicolumn{2}{|c|}{$\frac{\text { Nome do táxon }}{\text { FALCONIFORMES }}$}} \\
\hline & \\
\hline \multicolumn{2}{|l|}{ Falconidae } \\
\hline Ibycter americanus (Boddaert, 1783) & gralhão \\
\hline Caracara plancus (Miller, 1777) & caracará \\
\hline Milvago chimachima (Vieillot, 1816) & carrapateiro \\
\hline Milvago chimango (Vieillot, 1816) & chimango \\
\hline Herpetotheres cachinnans (Linnaeus, 1758) & acauã \\
\hline Micrastur ruficollis (Vieillot, 1817) & falcão-caburé \\
\hline Micrastur semitorquatus (Vieillot, 1817) & falcão-relógio \\
\hline Falco sparverius Linnaeus, 1758 & quiriquiri \\
\hline Falco rufigularis Daudin, 1800 & cauré \\
\hline Falco deiroleucus Temminck, 1825 & falcão-de-peito-laranja \\
\hline Falco femoralis Temminck, 1822 & falcão-de-coleira \\
\hline Falco peregrinus Tunstall, 1771 & falcão-peregrino \\
\hline \multicolumn{2}{|l|}{ EURYPYGIFORMES } \\
\hline \multicolumn{2}{|l|}{ Eurypygidae } \\
\hline Eurypyga helias (Pallas, 1781) & pavãozinho-do-pará \\
\hline \multicolumn{2}{|l|}{ GRUIFORMES } \\
\hline \multicolumn{2}{|l|}{ Aramidae } \\
\hline Aramus guarauna (Linnaeus, 1766) & carão \\
\hline \multicolumn{2}{|l|}{ Rallidae } \\
\hline [Coturnicops notatus (Gould, 1841)] & pinto-d'água-carijó \\
\hline Aramides ypecaha (Vieillot, 1819) & saracuruçu \\
\hline Aramides cajanea (Statius Muller, 1776) & saracura-três-potes \\
\hline [Aramides saracura $($ Spix, 1825)] & saracura-do-mato \\
\hline [Amaurolimnas concolor (Gosse, 1847)] & saracura-lisa \\
\hline Laterallus viridis (Statius Muller, 1776) & sanã-castanha \\
\hline Laterallus melanophaius (Vieillot, 1819) & sanã-parda \\
\hline Laterallus exilis (Temminck, 1831) & sanã-do-capim \\
\hline Porzana flaviventer (Boddaert, 1783) & sanã-amarela \\
\hline Porzana albicollis (Vieillot, 1819) & sanã-carijó \\
\hline Neocrex erythrops (Sclater, 1867) & turu-turu \\
\hline Pardirallus maculatus (Boddaert, 1783) & saracura-carijó \\
\hline Pardirallus nigricans (Vieillot, 1819) & saracura-sanã \\
\hline Gallinula galeata (Lichtenstein, 1850) & frango-d'água-comum \\
\hline Porphyrio martinica (Linnaeus, 1766) & frango-d'água-azul \\
\hline Porphyrio flavirostris (Gmelin, 1789) & frango-d'água-pequeno \\
\hline \multicolumn{2}{|l|}{ Heliornithidae } \\
\hline Heliornis fulica (Boddaert, 1783) & picaparra \\
\hline \multicolumn{2}{|l|}{ CARIAMIFORMES } \\
\hline \multicolumn{2}{|l|}{ Cariamidae } \\
\hline Cariama cristata (Linnaeus, 1766) & seriema \\
\hline \multicolumn{2}{|l|}{ CHARADRIIFORMES } \\
\hline \multicolumn{2}{|l|}{ Charadriidae } \\
\hline Vanellus cayanus (Latham, 1790) & batuíra-de-esporão \\
\hline Vanellus chilensis (Molina, 1782) & quero-quero \\
\hline Pluvialis dominica (Statius Muller, 1776) & batuiruçu \\
\hline [Pluvialis squatarola (Linnaeus, 1758)] & batuiruçu-de-axila-preta \\
\hline Charadrius collaris Vieillot, 1818 & batuíra-de-coleira \\
\hline [Charadrius semipalmatus Bonaparte, 1825] & batuíra-de-bando \\
\hline \multicolumn{2}{|l|}{ Recurvirostridae } \\
\hline Himantopus mexicanus (Statius Muller, 1776) & pernilongo-de-costas-negras \\
\hline Himantopus melanurus Vieillot, 1817 & pernilongo-de-costas-brancas \\
\hline \multicolumn{2}{|l|}{ Scolopacidae Rafinesque, 1815} \\
\hline Gallinago paraguaiae (Vieillot, 1816) & narceja \\
\hline Gallinago undulata (Boddaert, 1783) & narcejão \\
\hline Limosa haemastica (Linnaeus, 1758) & maçarico-de-bico-virado \\
\hline Bartramia longicauda (Bechstein, 1812) & maçarico-do-campo \\
\hline Actitis macularius (Linnaeus, 1766) & maçarico-pintado \\
\hline Tringa solitaria Wilson, 1813 & maçarico-solitário \\
\hline Tringa melanoleuca (Gmelin, 1789) & maçarico-grande-de-perna-amarela \\
\hline Tringa flavipes (Gmelin, 1789) & maçarico-de-perna-amarela \\
\hline Calidris fuscicollis (Vieillot, 1819) & maçarico-de-sobre-branco \\
\hline Calidris melanotos (Vieillot, 1819) & maçarico-de-colete \\
\hline Calidris himantopus (Bonaparte, 1826) & maçarico-pernilongo \\
\hline [Tryngites subruficollis (Vieillot, 1819)] & maçarico-acanelado \\
\hline
\end{tabular}


Tab. I. Cont.

\begin{tabular}{|c|c|}
\hline Nome do táxon & Nome popular \\
\hline Phalaropus tricolor (Vieillot, 1819) & pisa-n’água \\
\hline \multicolumn{2}{|l|}{ Jacanidae Chenu \& Des Murs, 1854} \\
\hline Jacana jacana (Linnaeus, 1766) & jaçanã \\
\hline \multicolumn{2}{|l|}{ Laridae } \\
\hline [Chroicocephalus cirrocephalus (Vieillot, 1818)] & gaivota-de-cabeça-cinza \\
\hline \multicolumn{2}{|l|}{ Sternidae } \\
\hline Sternula superciliaris (Vieillot, 1819) & trinta-réis-anão \\
\hline Phaetusa simplex (Gmelin, 1789) & trinta-réis-grande \\
\hline [Sterna trudeaui Audubon, 1838] & trinta-réis-de-coroa-branca \\
\hline \multicolumn{2}{|l|}{ Rynchopidae } \\
\hline Rynchops niger Linnaeus, 1758 & talha-mar \\
\hline \multicolumn{2}{|l|}{ COLUMBIFORMES } \\
\hline \multicolumn{2}{|l|}{ Columbidae } \\
\hline Columbina minuta (Linnaeus, 1766) & rolinha-de-asa-canela \\
\hline Columbina talpacoti (Temminck, 1811) & rolinha-roxa \\
\hline Columbina squammata (Lesson, 1831) & fogo-apagou \\
\hline Columbina picui (Temminck, 1813) & rolinha-picui \\
\hline Claravis pretiosa (Ferrari-Perez, 1886) & pararu-azul \\
\hline Uropelia campestris (Spix, 1825) & rolinha-vaqueira \\
\hline Columba livia Gmelin, 1789 & pombo-doméstico \\
\hline Patagioenas speciosa (Gmelin, 1789) & pombo-trocal \\
\hline Patagioenas picazuro (Temminck, 1813) & pombão \\
\hline Patagioenas cayennensis (Bonnaterre, 1792) & pomba-galega \\
\hline [Patagioenas plumbea (Vieillot, 1818)] & pomba-amargosa \\
\hline Zenaida auriculata (Des Murs, 1847) & pomba-de-bando \\
\hline Leptotila verreauxi Bonaparte, 1855 & juriti-pupu \\
\hline Leptotila rufaxilla (Richard \& Bernard, 1792) & juriti-gemedeira \\
\hline [Geotrygon montana (Linnaeus, 1758)] & pariri \\
\hline \multicolumn{2}{|l|}{ PSITTACIFORMES } \\
\hline \multicolumn{2}{|l|}{ Psittacidae } \\
\hline Anodorhynchus hyacinthinus (Latham, 1790) vu & arara-azul-grande \\
\hline Ara ararauna (Linnaeus, 1758) & arara-canindé \\
\hline Ara chloropterus Gray, 1859 & arara-vermelha-grande \\
\hline Orthopsittaca manilata (Boddaert, 1783) & maracanã-do-buriti \\
\hline Primolius maracana (Vieillot, 1816) & maracanã-verdadeira \\
\hline Primolius auricollis (Cassin, 1853) & maracanã-de-colar \\
\hline Diopsittaca nobilis (Linnaeus, 1758) & maracanã-pequena \\
\hline Aratinga acuticaudata (Vieillot, 1818) & aratinga-de-testa-azul \\
\hline Aratinga leucophthalma (Statius Muller, 1776) & periquitão-maracanã \\
\hline Aratinga nenday (Vieillot, 1823) & periquito-de-cabeça-preta \\
\hline Aratinga auricapillus (Kuhl, 1820) & jandaia-de-testa-vermelha \\
\hline Aratinga aurea (Gmelin, 1788) & periquito-rei \\
\hline Pyrrhura devillei (Massena \& Souancé, 1854) & tiriba-fogo \\
\hline Pyrrhura frontalis (Vieillot, 1817) & tiriba-de-testa-vermelha \\
\hline Pyrrhura molinae (Massena \& Souancé, 1854) & tiriba-de-cara-suja \\
\hline Myiopsitta monachus (Boddaert, 1783) & caturrita \\
\hline Forpus xanthopterygius (Spix, 1824) & tuim \\
\hline Brotogeris chiriri (Vieillot, 1818) & periquito-de-encontro-amarelo \\
\hline Alipiopsitta xanthops (Spix, 1824) & papagaio-galego \\
\hline Pionus menstruus (Linnaeus, 1766) & maitaca-de-cabeça-azul \\
\hline Pionus maximiliani (Kuhl, 1820) & maitaca-verde \\
\hline Amazona amazonica (Linnaeus, 1766) & curica \\
\hline Amazona aestiva (Linnaeus, 1758) & papagaio-verdadeiro \\
\hline \multicolumn{2}{|l|}{ CUCULIFORMES } \\
\hline \multicolumn{2}{|l|}{ Cuculidae } \\
\hline Coсcусиа minuta (Vieillot, 1817) & chincoã-pequeno \\
\hline Micrococcyx cinereus (Vieillot, 1817) & papa-lagarta-cinzento \\
\hline Piaya cayana (Linnaeus, 1766$)$ & alma-de-gato \\
\hline Coccyzus melacoryphus Vieillot, 1817 & papa-lagarta-acanelado \\
\hline Coccyzus americanus (Linnaeus, 1758) & papa-lagarta-de-asa-vermelha \\
\hline Coccyzus euleri Cabanis, 1873 & papa-lagarta-de-euler \\
\hline Crotophaga major Gmelin, 1788 & anu-coroca \\
\hline Crotophaga ani Linnaeus, 1758 & anu-preto \\
\hline Guira guira (Gmelin, 1788) & anu-branco \\
\hline Tapera naevia (Linnaeus, 1766) & saci \\
\hline Dromococcyx phasianellus (Spix, 1824) & peixe-frito-verdadeiro \\
\hline
\end{tabular}


Tab. I. Cont.

\begin{tabular}{|c|c|}
\hline Nome do táxon & Nome popular \\
\hline Dromococcyx pavoninus Pelzeln, 1870 & peixe-frito-pavonino \\
\hline \multicolumn{2}{|l|}{ STRIGIFORMES } \\
\hline \multicolumn{2}{|l|}{ Tytonidae } \\
\hline Tyto alba (Scopoli, 1769) & coruja-da-igreja \\
\hline \multicolumn{2}{|l|}{ Strigidae } \\
\hline Megascops choliba (Vieillot, 1817) & corujinha-do-mato \\
\hline Pulsatrix perspicillata $($ Latham, 1790$)$ & murucututu \\
\hline [Pulsatrix koeniswaldiana (Bertoni \& Bertoni, 1901)] & murucututu-de-barriga-amarela \\
\hline Bubo virginianus (Gmelin, 1788) & jacurutu \\
\hline$[$ Strix virgata $($ Cassin, 1849$)]$ & coruja-do-mato \\
\hline Strix huhula Daudin, 1800 & coruja-preta \\
\hline Glaucidium minutissimum (Wied, 1830) & caburé-miudinho \\
\hline Glaucidium brasilianum (Gmelin, 1788) & caburé \\
\hline Athene cunicularia (Molina, 1782) & coruja-buraqueira \\
\hline Asio clamator (Vieillot, 1808) & coruja-orelhuda \\
\hline Asio stygius (Wagler, 1832) & mocho-diabo \\
\hline Asio flammeus (Pontoppidan, 1763) & mocho-dos-banhados \\
\hline \multicolumn{2}{|l|}{ CAPRIMULGIFORMES } \\
\hline \multicolumn{2}{|l|}{ Nyctibiidae } \\
\hline Nyctibius grandis (Gmelin, 1789) & mãe-da-lua-gigante \\
\hline Nyctibius griseus (Gmelin, 1789) & mãe-da-lua \\
\hline \multicolumn{2}{|l|}{ Caprimulgidae } \\
\hline [Lurocalis semitorquatus (Gmelin, 1789)] & tuju \\
\hline Chordeiles pusillus Gould, 1861 & bacurauzinho \\
\hline Chordeiles acutipennis (Hermann, 1783) & bacurau-de-asa-fina \\
\hline Chordeiles minor (Forster, 1771) & bacurau-norte-americano \\
\hline Nyctiprogne leucopyga (Spix, 1825) & bacurau-de-cauda-barrada \\
\hline Podager nacunda (Vieillot, 1817) & corucão \\
\hline Nyctidromus albicollis (Gmelin, 1789) & bacurau \\
\hline [Nyctiphrynus ocellatus (Tschudi, 1844)] & bacurau-ocelado \\
\hline Caprimulgus rufus Boddaert, 1783 & joão-corta-pau \\
\hline Caprimulgus maculicaudus (Lawrence, 1862) & bacurau-de-rabo-maculado \\
\hline Caprimulgus parvulus Gould, 1837 & bacurau-chintã \\
\hline Hydropsalis torquata (Gmelin, 1789) & bacurau-tesoura \\
\hline [Eleothreptus candicans (Pelzeln, 1867)] $]^{\mathrm{EN}}$ & bacurau-de-rabo-branco \\
\hline \multicolumn{2}{|l|}{ APODIFORMES } \\
\hline \multicolumn{2}{|l|}{ Apodidae } \\
\hline Cypseloides senex (Temminck, 1826) & taperuçu-velho \\
\hline Streptoprocne zonaris (Shaw, 1796) & taperuçu-de-coleira-branca \\
\hline Chaetura meridionalis Hellmayr, 1907 & andorinhão-do-temporal \\
\hline Tachornis squamata (Cassin, 1853) & tesourinha \\
\hline \multicolumn{2}{|l|}{ Trochilidae } \\
\hline Phaethornis subochraceus Todd, 1915 & rabo-branco-de-barriga-fulva \\
\hline Phaethornis pretrei (Lesson \& Delattre, 1839) & rabo-branco-acanelado \\
\hline [Phaethornis eurynome (Lesson, 1832)] & rabo-branco-de-garganta-rajada \\
\hline Eupetomena macroura (Gmelin, 1788) & beija-flor-tesoura \\
\hline Aphantochroa cirrochloris (Vieillot, 1818) & beija-flor-cinza \\
\hline Florisuga fusca (Vieillot, 1817) & beija-flor-preto \\
\hline Colibri serrirostris (Vieillot, 1816) & beija-flor-de-orelha-violeta \\
\hline Anthracothorax nigricollis (Vieillot, 1817) & beija-flor-de-veste-preta \\
\hline Chrysolampis mosquitus (Linnaeus, 1758) & beija-flor-vermelho \\
\hline Lophornis gouldii (Lesson, 1832) & topetinho-do-brasil-central \\
\hline Lophornis magnificus (Vieillot, 1817) & topetinho-vermelho \\
\hline Chlorostilbon lucidus (Shaw, 1812) & besourinho-de-bico-vermelho \\
\hline Thalurania furcata (Gmelin, 1788) & beija-flor-tesoura-verde \\
\hline Thalurania glaucopis (Gmelin, 1788) & beija-flor-de-fronte-violeta \\
\hline Hylocharis cyanus (Vieillot, 1818) & beija-flor-roxo \\
\hline Hylocharis chrysura (Shaw, 1812) & beija-flor-dourado \\
\hline Leucochloris albicollis (Vieillot, 1818) & beija-flor-de-papo-branco \\
\hline Polytmus guainumbi (Pallas, 1764) & beija-flor-de-bico-curvo \\
\hline Amazilia versicolor (Vieillot, 1818) & beija-flor-de-banda-branca \\
\hline Amazilia fimbriata (Gmelin, 1788) & beija-flor-de-garganta-verde \\
\hline Heliactin bilophus (Temminck, 1820) & chifre-de-ouro \\
\hline Heliomaster squamosus (Temminck, 1823) & bico-reto-de-banda-branca \\
\hline Heliomaster furcifer (Shaw, 1812) & bico-reto-azul \\
\hline Calliphlox amethystina (Boddaert, 1783) & estrelinha-ametista \\
\hline
\end{tabular}


Tab. I. Cont.

\begin{tabular}{|c|c|}
\hline Nome do táxon & Nome popular \\
\hline \multicolumn{2}{|l|}{ TROGONIFORMES } \\
\hline \multicolumn{2}{|l|}{ Trogonidae } \\
\hline Trogon surrucura Vieillot, 1817 & surucuá-variado \\
\hline Trogon curucui Linnaeus, 1766 & surucuá-de-barriga-vermelha \\
\hline Trogon rufus Gmelin, 1788 & surucuá-de-barriga-amarela \\
\hline \multicolumn{2}{|l|}{ CORACIIFORMES } \\
\hline \multicolumn{2}{|l|}{ Alcedinidae } \\
\hline Megaceryle torquata (Linnaeus, 1766) & martim-pescador-grande \\
\hline Chloroceryle amazona (Latham, 1790) & martim-pescador-verde \\
\hline Chloroceryle aenea (Pallas, 1764) & martinho \\
\hline Chloroceryle americana (Gmelin, 1788) & martim-pescador-pequeno \\
\hline Chloroceryle inda (Linnaeus, 1766) & martim-pescador-da-mata \\
\hline \multicolumn{2}{|l|}{ Momotidae } \\
\hline Baryphthengus ruficapillus (Vieillot, 1818) & juruva-verde \\
\hline Momotus momota (Linnaeus, 1766) & udu-de-coroa-azul \\
\hline \multicolumn{2}{|l|}{ GALBULIFORMES } \\
\hline \multicolumn{2}{|l|}{ Galbulidae } \\
\hline Brachygalba lugubris (Swainson, 1838) & ariramba-preta \\
\hline Galbula ruficauda Cuvier, 1816 & ariramba-de-cauda-ruiva \\
\hline \multicolumn{2}{|l|}{ Bucconidae } \\
\hline Notharchus swainsoni (Gray, 1846) & macuru-de-barriga-castanha \\
\hline [Bucco tamatia Gmelin, 1788] & rapazinho-carijó \\
\hline Nystalus chacuru (Vieillot, 1816) & joão-bobo \\
\hline Nystalus maculatus (Gmelin, 1788) & rapazinho-dos-velhos \\
\hline Nystalus striatipectus (Sclater, 1854) & rapazinho-do-chaco \\
\hline Nonnula rubecula (Spix, 1824) & macuru \\
\hline Monasa nigrifrons (Spix, 1824) & chora-chuva-preto \\
\hline Chelidoptera tenebrosa (Pallas, 1782) & urubuzinho \\
\hline \multicolumn{2}{|l|}{ PICIFORMES } \\
\hline \multicolumn{2}{|l|}{ Ramphastidae } \\
\hline Ramphastos toco Statius Muller, 1776 & tucanuçu \\
\hline Ramphastos vitellinus Lichtenstein, 1823 & tucano-de-bico-preto \\
\hline Ramphastos dicolorus Linnaeus, 1766 & tucano-de-bico-verde \\
\hline Pteroglossus castanotis Gould, 1834 & aracari-castanho \\
\hline \multicolumn{2}{|l|}{ Picidae } \\
\hline Picumnus cirratus Temminck, 1825 & pica-pau-anão-barrado \\
\hline Picumnus albosquamatus d'Orbigny, 1840 & pica-pau-anão-escamado \\
\hline Melanerpes candidus (Otto, 1796) & birro \\
\hline Melanerpes flavifrons (Vieillot, 1818) & benedito-de-testa-amarela \\
\hline Melanerpes cactorum (d'Orbigny, 1840) & pica-pau-de-testa-branca \\
\hline Veniliornis passerinus (Linnaeus, 1766) & picapauzinho-anão \\
\hline [Veniliornis spilogaster (Wagler, 1827)] & picapauzinho-verde-carijó \\
\hline Veniliornis mixtus (Boddaert, 1783) & pica-pau-chorão \\
\hline Piculus chrysochloros (Vieillot, 1818) & pica-pau-dourado-escuro \\
\hline Colaptes melanochloros (Gmelin, 1788) & pica-pau-verde-barrado \\
\hline Colaptes campestris (Vieillot, 1818) & pica-pau-do-campo \\
\hline Celeus lugubris (Malherbe, 1851) & pica-pau-louro \\
\hline Celeus flavescens (Gmelin, 1788) & pica-pau-de-cabeça-amarela \\
\hline Celeus flavus (Statius Muller, 1776) & pica-pau-amarelo \\
\hline Dryocopus lineatus (Linnaeus, 1766) & pica-pau-de-banda-branca \\
\hline Campephilus robustus (Lichtenstein, 1818) & pica-pau-rei \\
\hline Campephilus melanoleucos (Gmelin, 1788) & pica-pau-de-topete-vermelho \\
\hline Campephilus leucopogon (Valenciennes, 1826) & pica-pau-de-barriga-preta \\
\hline \multicolumn{2}{|l|}{ PASSERIFORMES } \\
\hline \multicolumn{2}{|l|}{ Thamnophilidae } \\
\hline Terenura maculata (Wied, 1831) & zidedê \\
\hline Myrmorchilus strigilatus (Wied, 1831) & piu-piu \\
\hline Myrmotherula multostriata Sclater, 1858 & choquinha-estriada-da-amazônia \\
\hline Formicivora melanogaster Pelzeln, 1868 & formigueiro-de-barriga-preta \\
\hline Formicivora rufa (Wied, 1831) & papa-formiga-vermelho \\
\hline Dysithamnus mentalis (Temminck, 1823) & choquinha-lisa \\
\hline Herpsilochmus atricapillus Pelzeln, 1868 & chorozinho-de-chapéu-preto \\
\hline Herpsilochmus longirostris Pelzeln, 1868 & chorozinho-de-bico-comprido \\
\hline Thamnophilus doliatus (Linnaeus, 1764) & choca-barrada \\
\hline [Thamnophilus ruficapillus Vieillot, 1816] & choca-de-chapéu-vermelho \\
\hline Thamnophilus torquatus Swainson, 1825 & choca-de-asa-vermelha \\
\hline
\end{tabular}


Tab. I. Cont.

\begin{tabular}{|c|c|}
\hline Nome do táxon & Nome popular \\
\hline Thamnophilus sticturus Pelzeln, 1868 & choca-da-bolívia \\
\hline Thamnophilus pelzelni Hellmayr, 1924 & choca-do-planalto \\
\hline Thamnophilus caerulescens Vieillot, 1816 & choca-da-mata \\
\hline Taraba major (Vieillot, 1816) & choró-boi \\
\hline Hypoedaleus guttatus (Vieillot, 1816) & chocão-carijó \\
\hline Pyriglena leuconota (Spix, 1824) & papa-taoca \\
\hline Pyriglena leucoptera (Vieillot, 1818) & papa-taoca-do-sul \\
\hline Cercomacra melanaria (Ménétriès, 1835) & chororó-do-pantanal \\
\hline \multicolumn{2}{|l|}{ Melanopareiidae } \\
\hline Melanopareia torquata (Wied, 1831) & tapaculo-de-colarinho \\
\hline \multicolumn{2}{|l|}{ Conopophagidae } \\
\hline Conopophaga lineata (Wied, 1831) & chupa-dente \\
\hline \multicolumn{2}{|l|}{ Formicariidae } \\
\hline Chamaeza campanisona (Lichtenstein, 1823) & tovaca-campainha \\
\hline \multicolumn{2}{|l|}{ Scleruridae } \\
\hline [Sclerurus scansor (Ménétriès, 1835)] & vira-folha \\
\hline Geositta poeciloptera (Wied, 1830) $\mathrm{vU}$ & andarilho \\
\hline \multicolumn{2}{|l|}{ Dendrocolaptidae } \\
\hline Sittasomus griseicapillus (Vieillot, 1818) & arapaçu-verde \\
\hline Xiphorhynchus guttatus (Lichtenstein, 1820) & arapaçu-de-garganta-amarela \\
\hline Campylorhamphus trochilirostris (Lichtenstein, 1820) & arapaçu-beija-flor \\
\hline Dendroplex picus (Gmelin, 1788) & arapaçu-de-bico-branco \\
\hline Lepidocolaptes angustirostris (Vieillot, 1818) & arapaçu-do-cerrado \\
\hline Dendrocolaptes picumnus Lichtenstein, 1820 & arapaçu-meio-barrado \\
\hline Dendrocolaptes platyrostris Spix, 1825 & arapaçu-grande \\
\hline Xiphocolaptes albicollis (Vieillot, 1818) & arapaçu-de-garganta-branca \\
\hline Xiphocolaptes major (Vieillot, 1818) & arapaçu-do-campo \\
\hline \multicolumn{2}{|l|}{ Furnariidae } \\
\hline [Xenops minutus (Sparrman, 1788)] & bico-virado-miúdo \\
\hline Xenops rutilans Temminck, 1821 & bico-virado-carijó \\
\hline Furnarius leucopus Swainson, 1838 & casaca-de-couro-amarelo \\
\hline Furnarius rufus (Gmelin, 1788) & joão-de-barro \\
\hline [Lochmias nematura (Lichtenstein, 1823)] & joão-porca \\
\hline Automolus leucophthalmus (Wied, 1821) & barranqueiro-de-olho-branco \\
\hline Hylocryptus rectirostris (Wied, 1831) & fura-barreira \\
\hline Philydor lichtensteini Cabanis \& Heine, 1859 & limpa-folha-ocráceo \\
\hline Philydor rufum (Vieillot, 1818) & limpa-folha-de-testa-baia \\
\hline Syndactyla rufosuperciliata (Lafresnaye, 1832) & trepador-quiete \\
\hline Syndactyla dimidiata (Pelzeln, 1859) & limpa-folha-do-brejo \\
\hline Pseudoseisura unirufa (d'Orbigny \& Lafresnaye, 1838) & casaca-de-couro-de-crista-cinza \\
\hline Phacellodomus rufifrons (Wied, 1821) & joão-de-pau \\
\hline Phacellodomus ruber (Vieillot, 1817) & graveteiro \\
\hline Anumbius annumbi (Vieillot, 1817) & cochicho \\
\hline Schoeniophylax phryganophilus (Vieillot, 1817) & bichoita \\
\hline Certhiaxis cinnamomeus (Gmelin, 1788) & curutié \\
\hline Synallaxis ruficapilla Vieillot, 1819 & pichororé \\
\hline Synallaxis cinerascens Temminck, 1823 & pi-puí \\
\hline Synallaxis frontalis Pelzeln, 1859 & petrim \\
\hline Synallaxis albescens Temminck, 1823 & uí-pi \\
\hline [Synallaxis spixi Sclater, 1856] & joão-teneném \\
\hline Synallaxis hypospodia Sclater, 1874 & joão-grilo \\
\hline Synallaxis albilora Pelzeln, 1856 & joão-do-pantanal \\
\hline Synallaxis scutata Sclater, 1859 & estrelinha-preta \\
\hline Cranioleuca vulpina (Pelzeln, 1856) & arredio-do-rio \\
\hline \multicolumn{2}{|l|}{ Pipridae } \\
\hline Neopelma pallescens (Lafresnaye, 1853) & fruxu-do-cerradão \\
\hline Pipra fasciicauda Hellmayr, 1906 & uirapuru-laranja \\
\hline Manacus manacus (Linnaeus, 1766) & rendeira \\
\hline Chiroxiphia caudata (Shaw \& Nodder, 1793) & tangará \\
\hline Antilophia galeata (Lichtenstein, 1823) & soldadinho \\
\hline [Machaeropterus pyrocephalus (Sclater, 1852)] & uirapuru-cigarra \\
\hline \multicolumn{2}{|l|}{ Tityridae } \\
\hline [Oxyruncus cristatus Swainson, 1821] & araponga-do-horto \\
\hline Schiffornis virescens (Lafresnaye, 1838) & flautim \\
\hline Tityra inquisitor (Lichtenstein, 1823) & anambé-branco-de-bochecha-parda \\
\hline Tityra cayana (Linnaeus, 1766 ) & anambé-branco-de-rabo-preto \\
\hline
\end{tabular}


Tab. I. Cont.

\begin{tabular}{|c|c|}
\hline Nome do táxon & Nome popular \\
\hline Tityra semifasciata (Spix, 1825) & anambé-branco-de-máscara-negra \\
\hline Pachyramphus viridis (Vieillot, 1816) & caneleiro-verde \\
\hline [Pachyramphus castaneus (Jardine \& Selby, 1827)] & caneleiro \\
\hline Pachyramphus polychopterus (Vieillot, 1818) & caneleiro-preto \\
\hline [Pachyramphus marginatus (Lichtenstein, 1823)] & caneleiro-bordado \\
\hline Pachyramphus validus (Lichtenstein, 1823) & caneleiro-de-chapéu-preto \\
\hline Xenopsaris albinucha (Burmeister, 1869) & tijerila \\
\hline \multicolumn{2}{|l|}{ Cotingidae } \\
\hline Procnias nudicollis (Vieillot, 1817) & araponga \\
\hline Pyroderus scutatus (Shaw, 1792) & pavó \\
\hline Phibalura flavirostris Vieillot, 1816 & tesourinha-da-mata \\
\hline \multicolumn{2}{|l|}{ Rynchocyclidae } \\
\hline Mionectes rufiventris Cabanis, 1846 & abre-asa-de-cabeça-cinza \\
\hline Leptopogon amaurocephalus Tschudi, 1846 & cabeçudo \\
\hline Corythopis delalandi (Lesson, 1830) & estalador \\
\hline Phylloscartes ventralis (Temminck, 1824) & borboletinha-do-mato \\
\hline Phylloscartes paulista Ihering \& Ihering, 1907 & não-pode-parar \\
\hline Tolmomyias sulphurescens (Spix, 1825) & bico-chato-de-orelha-preta \\
\hline Todirostrum cinereum (Linnaeus, 1766) & ferreirinho-relógio \\
\hline Poecilotriccus plumbeiceps (Lafresnaye, 1846) & tororó \\
\hline Poecilotriccus latirostris (Pelzeln, 1868) & ferreirinho-de-cara-parda \\
\hline Myiornis auricularis (Vieillot, 1818) & miudinho \\
\hline Hemitriccus striaticollis (Lafresnaye, 1853) & sebinho-rajado-amarelo \\
\hline Hemitriccus margaritaceiventer (d'Orbigny \& Lafresnaye, 1837) & sebinho-de-olho-de-ouro \\
\hline \multicolumn{2}{|l|}{ Tyrannidae } \\
\hline Hirundinea ferruginea (Gmelin, 1788) & gibão-de-couro \\
\hline Inezia inornata (Salvadori, 1897) & alegrinho-do-chaco \\
\hline Euscarthmus meloryphus Wied, 1831 & barulhento \\
\hline Euscarthmus rufomarginatus (Pelzeln, 1868) & maria-corruíra \\
\hline Camptostoma obsoletum (Temminck, 1824) & risadinha \\
\hline Elaenia flavogaster (Thunberg, 1822) & guaracava-de-barriga-amarela \\
\hline Elaenia spectabilis Pelzeln, 1868 & guaracava-grande \\
\hline Elaenia chilensis Hellmayr, 1927 & guaracava-de-crista-branca \\
\hline Elaenia parvirostris Pelzeln, 1868 & guaracava-de-bico-curto \\
\hline Elaenia mesoleuca (Deppe, 1830) & tuque \\
\hline Elaenia cristata Pelzeln, 1868 & guaracava-de-topete-uniforme \\
\hline Elaenia chiriquensis Lawrence, 1865 & chibum \\
\hline Elaenia obscura (d'Orbigny \& Lafresnaye, 1837) & tucão \\
\hline Suiriri suiriri (Vieillot, 1818) & suiriri-cinzento \\
\hline Suiriri islerorum Zimmer, Whittaker \& Oren, 2001 & suiriri-da-chapada \\
\hline Myiopagis gaimardii (d'Orbigny, 1839) & maria-pechim \\
\hline Myiopagis caniceps (Swainson, 1835) & guaracava-cinzenta \\
\hline Myiopagis viridicata (Vieillot, 1817) & guaracava-de-crista-alaranjada \\
\hline Capsiempis flaveola (Lichtenstein, 1823) & marianinha-amarela \\
\hline Phaeomyias murina (Spix, 1825) & bagageiro \\
\hline Phyllomyias virescens (Temminck, 1824) & piolhinho-verdoso \\
\hline Phyllomyias reiseri Hellmayr, 1905 & piolhinho-do-grotão \\
\hline Phyllomyias fasciatus (Thunberg, 1822) & piolhinho \\
\hline Culicivora caudacuta (Vieillot, 1818) ${ }^{\mathrm{vu}}$ & papa-moscas-do-campo \\
\hline Polystictus pectoralis (Vieillot, 1817) $\mathrm{vu}$ & papa-moscas-canela \\
\hline Pseudocolopteryx sclateri (Oustalet, 1892) & tricolino \\
\hline Pseudocolopteryx acutipennis (Sclater \& Salvin, 1873) & tricolino-oliváceo \\
\hline [Pseudocolopteryx dinelliana Lillo, 1905] & tricolino-pardo \\
\hline [Serpophaga nigricans (Vieillot, 1817)] & joão-pobre \\
\hline Serpophaga subcristata (Vieillot, 1817) & alegrinho \\
\hline [Serpophaga griseicapilla Straneck, 2007] & alegrinho-trinador \\
\hline Serpophaga munda Berlepsch, 1893 & alegrinho-de-barriga-branca \\
\hline Platyrinchus mystaceus Vieillot, 1818 & patinho \\
\hline Attila phoenicurus Pelzeln, 1868 & capitão-castanho \\
\hline [Attila bolivianus Lafresnaye, 1848] & bate-pára \\
\hline Legatus leucophaius (Vieillot, 1818) & bem-te-vi-pirata \\
\hline Myiarchus tuberculifer (d'Orbigny \& Lafresnaye, 1837) & maria-cavaleira-pequena \\
\hline Myiarchus swainsoni Cabanis \& Heine, 1859 & irré \\
\hline Myiarchus ferox (Gmelin, 1789) & maria-cavaleira \\
\hline Myiarchus tyrannulus (Statius Muller, 1776) & maria-cavaleira-de-rabo-enferrujado \\
\hline Sirystes sibilator (Vieillot, 1818) & gritador \\
\hline
\end{tabular}


Tab. I. Cont.

\begin{tabular}{|c|c|}
\hline Nome do táxon & Nome popular \\
\hline Casiornis rufus (Vieillot, 1816) & caneleiro \\
\hline Pitangus sulphuratus (Linnaeus, 1766) & bem-te-vi \\
\hline Philohydor lictor (Lichtenstein, 1823) & bentevizinho-do-brejo \\
\hline Machetornis rixosa (Vieillot, 1819) & suiriri-cavaleiro \\
\hline Myiodynastes maculatus (Statius Muller, 1776) & bem-te-vi-rajado \\
\hline Tyrannopsis sulphurea (Spix, 1825) & suiriri-de-garganta-rajada \\
\hline Megarynchus pitangua (Linnaeus, 1766) & neinei \\
\hline Myiozetetes cayanensis (Linnaeus, 1766) & bentevizinho-de-asa-ferrugínea \\
\hline Myiozetetes similis (Spix, 1825) & bentevizinho-de-penacho-vermelho \\
\hline Tyrannus albogularis Burmeister, 1856 & suiriri-de-garganta-branca \\
\hline Tyrannus melancholicus Vieillot, 1819 & suiriri \\
\hline Tyrannus savana Vieillot, 1808 & tesourinha \\
\hline Tyrannus tyrannus (Linnaeus, 1766) & suiriri-valente \\
\hline \multicolumn{2}{|c|}{ Griseotyrannus aurantioatrocristatus (d'Orbigny \& Lafresnaye, 1837)peitica-de-chapéu-preto } \\
\hline Empidonomus varius (Vieillot, 1818) & peitica \\
\hline Colonia colonus (Vieillot, 1818) & viuvinha \\
\hline Myiophobus fasciatus (Statius Muller, 1776) & filipe \\
\hline Sublegatus modestus (Wied, 1831) & guaracava-modesta \\
\hline Pyrocephalus rubinus (Boddaert, 1783) & príncipe \\
\hline Fluvicola albiventer (Spix, 1825) & lavadeira-de-cara-branca \\
\hline Fluvicola nengeta (Linnaeus, 1766) & lavadeira-mascarada \\
\hline Arundinicola leucocephala (Linnaeus, 1764) & freirinha \\
\hline Gubernetes yetapa (Vieillot, 1818) & tesoura-do-brejo \\
\hline Alectrurus tricolor (Vieillot, 1816) ${ }^{\mathrm{vu}}$ & galito \\
\hline Alectrurus risora (Vieillot, 1824) & tesoura-do-campo \\
\hline Cnemotriccus fuscatus (Wied, 1831) & guaracavuçu \\
\hline Lathrotriccus euleri (Cabanis, 1868) & enferrujado \\
\hline Empidonax alnorum Brewster, 1895 & papa-moscas-de-alder \\
\hline Contopus cinereus (Spix, 1825) & papa-moscas-cinzento \\
\hline Knipolegus striaticeps (d'Orbigny \& Lafresnaye, 1837) & maria-preta-acinzentada \\
\hline [Knipolegus hudsoni Sclater, 1872] & maria-preta-do-sul \\
\hline Knipolegus cyanirostris (Vieillot, 1818) & maria-preta-de-bico-azulado \\
\hline [Knipolegus aterrimus Kaup, 1853] & maria-preta \\
\hline Knipolegus lophotes Boie, 1828 & maria-preta-de-penacho \\
\hline Hymenops perspicillatus (Gmelin, 1789) & viuvinha-de-óculos \\
\hline Satrapa icterophrys (Vieillot, 1818) & suiriri-pequeno \\
\hline Xolmis cinereus (Vieillot, 1816) & primavera \\
\hline Xolmis velatus (Lichtenstein, 1823) & noivinha-branca \\
\hline Xolmis irupero (Vieillot, 1823) & noivinha \\
\hline \multicolumn{2}{|l|}{ Vireonidae } \\
\hline Cyclarhis gujanensis (Gmelin, 1789) & pitiguari \\
\hline Vireo olivaceus (Linnaeus, 1766) & juruviara \\
\hline Hylophilus poicilotis Temminck, 1822 & verdinho-coroado \\
\hline Hylophilus amaurocephalus (Nordamann, 1835) & vite-vite-de-olho-cinza \\
\hline Hylophilus pectoralis Sclater, 1866 & vite-vite-de-cabeça-cinza \\
\hline \multicolumn{2}{|l|}{ Corvidae } \\
\hline Cyanocorax cyanomelas (Vieillot, 1818) & gralha-do-pantanal \\
\hline Cyanocorax cristatellus (Temminck, 1823) & gralha-do-cerrado \\
\hline Cyanocorax chrysops (Vieillot, 1818) & gralha-picaça \\
\hline Cyanocorax cyanopogon (Wied, 1821) & gralha-cancã \\
\hline \multicolumn{2}{|l|}{ Hirundinidae } \\
\hline [Pygochelidon cyanoleuca (Vieillot, 1817)] & andorinha-pequena-de-casa \\
\hline Alopochelidon fucata (Temminck, 1822) & andorinha-morena \\
\hline Stelgidopteryx ruficollis (Vieillot, 1817) & andorinha-serradora \\
\hline Progne tapera (Vieillot, 1817) & andorinha-do-campo \\
\hline Progne chalybea (Gmelin, 1789) & andorinha-doméstica-grande \\
\hline Tachycineta albiventer (Boddaert, 1783) & andorinha-do-rio \\
\hline Tachycineta leucorrhoa (Vieillot, 1817) & andorinha-de-sobre-branco \\
\hline Riparia riparia (Linnaeus, 1758) & andorinha-do-barranco \\
\hline Hirundo rustica Linnaeus, 1758 & andorinha-de-bando \\
\hline Petrochelidon pyrrhonota (Vieillot, 1817) & andorinha-de-dorso-acanelado \\
\hline \multicolumn{2}{|l|}{ Troglodytidae } \\
\hline Troglodytes musculus Naumann, 1823 & corruíra \\
\hline [Cistothorus platensis (Latham, 1790)] & corruíra-do-campo \\
\hline Campylorhynchus turdinus (Wied, 1831) & catatau \\
\hline [Pheugopedius genibarbis (Swainson, 1838)] & garrinchão-pai-avô \\
\hline
\end{tabular}


Tab. I. Cont.

\begin{tabular}{|c|c|}
\hline Nome do táxon & Nome popular \\
\hline Cantorchilus leucotis (Lafresnaye, 1845) & garrinchão-de-barriga-vemelha \\
\hline Cantorchilus guarayanus (d’Orbigny \& Lafresnaye, 1837) & garrincha-do-oeste \\
\hline \multicolumn{2}{|l|}{ Donacobiidae } \\
\hline Donacobius atricapilla (Linnaeus, 1766) & japacanim \\
\hline \multicolumn{2}{|l|}{ Polioptilidae } \\
\hline Polioptila dumicola (Vieillot, 1817) & balança-rabo-de-máscara \\
\hline \multicolumn{2}{|l|}{ Turdidae } \\
\hline Catharus fuscescens (Stephens, 1817) & sabiá-norte-americano \\
\hline Turdus rufiventris Vieillot, 1818 & sabiá-laranjeira \\
\hline Turdus leucomelas Vieillot, 1818 & sabiá-barranco \\
\hline Turdus amaurochalinus Cabanis, 1850 & sabiá-poca \\
\hline [Turdus subalaris (Seebohm, 1887)] & sabiá-ferreiro \\
\hline Turdus albicollis Vieillot, 1818 & sabiá-coleira \\
\hline \multicolumn{2}{|l|}{ Mimidae } \\
\hline Mimus saturninus (Lichtenstein, 1823) & sabiá-do-campo \\
\hline Mimus triurus (Vieillot, 1818) & calhandra-de-três-rabos \\
\hline \multicolumn{2}{|l|}{ Motacillidae } \\
\hline Anthus lutescens Pucheran, 1855 & caminheiro-zumbidor \\
\hline \multicolumn{2}{|l|}{ Coerebidae } \\
\hline Coereba flaveola (Linnaeus, 1758) & cambacica \\
\hline \multicolumn{2}{|l|}{ Thraupidae } \\
\hline Saltator maximus (Statius Muller, 1776) & tempera-viola \\
\hline Saltator coerulescens Vieillot, 1817 & sabiá-congá \\
\hline Saltator similis d'Orbigny \& Lafresnaye, 1837 & trinca-ferro-verdadeiro \\
\hline Saltator aurantiirostris Vieillot, 1817 & bico-duro \\
\hline Saltatricula atricollis Vieillot, 1817 & bico-de-pimenta \\
\hline Conothraupis mesoleuca (Berlioz, 1939) & tiê-bicudo \\
\hline Schistochlamys melanopis (Latham, 1790) & sanhaçu-de-coleira \\
\hline Schistochlamys ruficapillus (Vieillot, 1817) & bico-de-veludo \\
\hline Cissopis leverianus (Gmelin, 1788) & tietinga \\
\hline Neothraupis fasciata (Lichtenstein, 1823) & cigarra-do-campo \\
\hline Nemosia pileata (Boddaert, 1783) & saíra-de-chapéu-preto \\
\hline Thlypopsis sordida (d'Orbigny \& Lafresnaye, 1837) & saí-canário \\
\hline Cypsnagra hirundinacea (Lesson, 1831) & bandoleta \\
\hline Trichothraupis melanops (Vieillot, 1818) & tiê-de-topete \\
\hline Eucometis penicillata (Spix, 1825) & pipira-da-taoca \\
\hline Tachyphonus coronatus (Vieillot, 1822) & tiê-preto \\
\hline Tachyphonus rufus (Boddaert, 1783) & pipira-preta \\
\hline Ramphocelus carbo (Pallas, 1764) & pipira-vermelha \\
\hline Thraupis sayaca (Linnaeus, 1766) & sanhaçu-cinzento \\
\hline Thraupis palmarum (Wied, 1823$)$ & sanhaçu-do-coqueiro \\
\hline Pipraeidea melanonota (Vieillot, 1819) & saíra-viúva \\
\hline Pipraeidea bonariensis (Gmelin, 1789) & sanhaçu-papa-laranja \\
\hline Tangara seledon (Statius Muller, 1776) & saíra-sete-cores \\
\hline Tangara cayana (Linnaeus, 1766) & saíra-amarela \\
\hline Tersina viridis (Illiger, 1811) & saí-andorinha \\
\hline Dacnis cayana (Linnaeus, 1766) & saí-azul \\
\hline Cyanerpes cyaneus (Linnaeus, 1766) & saíra-beija-flor \\
\hline Hemithraupis guira (Linnaeus, 1766) & saíra-de-papo-preto \\
\hline Conirostrum speciosum (Temminck, 1824) & figuinha-de-rabo-castanho \\
\hline \multicolumn{2}{|l|}{ Emberizidae } \\
\hline Zonotrichia capensis (Statius Muller, 1776) & tico-tico \\
\hline Ammodramus humeralis (Bosc, 1792) & tico-tico-do-campo \\
\hline Porphyrospiza caerulescens (Wied, 1830) & campainha-azul \\
\hline Donacospiza albifrons (Vieillot, 1817) & tico-tico-do-banhado \\
\hline Poospiza melanoleuca (d'Orbigny \& Lafresnaye, 1837) & capacetinho \\
\hline Poospiza cinerea Bonnaparte, 1850 & capacetinho-do-oco-do-pau \\
\hline [Sicalis citrina Pelzeln, 1870] & canário-rasteirinho \\
\hline Sicalis flaveola (Linnaeus, 1766) & canário-da-terra-verdadeiro \\
\hline Sicalis luteola (Sparrman, 1789) & tipio \\
\hline Emberizoides herbicola (Vieillot, 1817) & canário-do-campo \\
\hline Emberizoides ypiranganus Ihering \& Ihering, 1907 & canário-do-brejo \\
\hline Embernagra platensis (Gmelin, 1789) & sabiá-do-banhado \\
\hline Volatinia jacarina (Linnaeus, 1766) & tiziu \\
\hline 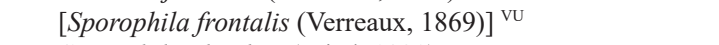 & pixoxó \\
\hline Sporophila plumbea (Wied, 1830) & patativa \\
\hline
\end{tabular}


Tab. I. Cont.

\begin{tabular}{|c|c|}
\hline Nome do táxon & Nome popular \\
\hline Sporophila collaris (Boddaert, 1783) & coleiro-do-brejo \\
\hline Sporophila lineola (Linnaeus, 1758) & bigodinho \\
\hline Sporophila nigricollis (Vieillot, 1823) & baiano \\
\hline Sporophila caerulescens (Vieillot, 1823) & coleirinho \\
\hline Sporophila leucoptera (Vieillot, 1817) & chorão \\
\hline Sporophila nigrorufa (d'Orbigny \& Lafresnaye, 1837) vu & caboclinho-do-sertão \\
\hline Sporophila bouvreuil (Statius Muller, 1776) & caboclinho \\
\hline Sporophila hypoxantha Cabanis, 1851 & caboclinho-de-barriga-vermelha \\
\hline Sporophila ruficollis Cabanis, 1851 & caboclinho-de-papo-escuro \\
\hline Sporophila palustris (Barrows, 1883) $\mathrm{EN}$ & caboclinho-de-papo-branco \\
\hline Sporophila hypochroma Todd, 1915 & caboclinho-de-sobre-ferrugem \\
\hline Sporophila cinnamomea (Lafresnaye, 1839) vu & caboclinho-de-chapéu-cinzento \\
\hline [Sporophila melanogaster (Pelzeln, 1870)] vu & caboclinho-de-barriga-preta \\
\hline Sporophila angolensis (Linnaeus, 1766) & curió \\
\hline$[\text { Sporophila maximiliani }(\text { Cabanis, } 1851)]^{\mathrm{CR}}$ & bicudo \\
\hline Tiaris obscurus (d’Orbigny \& Lafresnaye, 1837) & cigarra-parda \\
\hline Arremon taciturnus (Hermann, 1783) & tico-tico-de-bico-preto \\
\hline Arremon flavirostris Swainson, 1838 & tico-tico-de-bico-amarelo \\
\hline Coryphospingus cucullatus (Statius Muller, 1776) & tico-tico-rei \\
\hline Paroaria coronata (Miller, 1776) & cardeal \\
\hline Paroaria capitata (d'Orbigny \& Lafresnaye, 1837) & cavalaria \\
\hline Charitospiza eucosma Oberholser, 1905 & mineirinho \\
\hline Coryphaspiza melanotis (Temminck, 1822) $\mathrm{vu}$ & tico-tico-de-máscara-negra \\
\hline \multicolumn{2}{|l|}{ Cardinalidae } \\
\hline Piranga flava (Vieillot, 1822) & sanhaçu-de-fogo \\
\hline Habia rubica (Vieillot, 1817) & tiê-do-mato-grosso \\
\hline Pheucticus aureoventris (d'Orbigny \& Lafresnaye, 1837) & rei-do-bosque \\
\hline Cyanoloxia moesta (Hartlaub, 1853) & negrinho-do-mato \\
\hline Cyanoloxia cyanoides (Lafresnaye, 1847) & azulão-da-amazônia \\
\hline Cyanoloxia brissonii (Lichtenstein, 1823) & azulão \\
\hline Cyanoloxia glaucocerulea (d'Orbigny \& Lafresnaye, 1837) & azulinho \\
\hline \multicolumn{2}{|l|}{ Parulidae } \\
\hline Parula pitiayumi (Vieillot, 1817) & mariquita \\
\hline Geothlypis aequinoctialis (Gmelin, 1789) & pia-cobra \\
\hline Basileuterus culicivorus (Deppe, 1830) & pula-pula \\
\hline Basileuterus hypoleucus Bonaparte, 1830 & pula-pula-de-barriga-branca \\
\hline Basileuterus flaveolus (Baird, 1865) & canário-do-mato \\
\hline Basileuterus leucoblepharus (Vieillot, 1817) & pula-pula-assobiador \\
\hline Basileuterus leucophrys Pelzeln, 1868 & pula-pula-de-sobrancelha \\
\hline \multicolumn{2}{|l|}{ Icteridae } \\
\hline Psarocolius decumanus (Pallas, 1769) & japu \\
\hline Procacicus solitarius (Vieillot, 1816) & iraúna-de-bico-branco \\
\hline Cacicus chrysopterus (Vigors, 1825) & tecelão \\
\hline Cacicus haemorrhous (Linnaeus, 1766) & guaxe \\
\hline Cacicus cela (Linnaeus, 1758) & xexéu \\
\hline Icterus cayanensis (Linnaeus, 1766) & inhapim \\
\hline Icterus pyrrhopterus (Vieillot, 1819) & encontro \\
\hline Icterus periporphyrus (Bonaparte, 1850 ) & pega \\
\hline Icterus croconotus (Wagler, 1829) & joão-pinto \\
\hline Gnorimopsar chopi (Vieillot, 1819) & graúna \\
\hline Amblyramphus holosericeus (Scopoli, 1786) & cardeal-do-banhado \\
\hline Agelasticus cyanopus (Vieillot, 1819) & carretão \\
\hline Chrysomus ruficapillus (Vieillot, 1819) & garibaldi \\
\hline Pseudoleistes guirahuro (Vieillot, 1819) & chopim-do-brejo \\
\hline Agelaioides badius (Vieillot, 1819) & asa-de-telha \\
\hline Molothrus rufoaxillaris Cassin, 1866 & vira-bosta-picumã \\
\hline Molothrus oryzivorus (Gmelin, 1788) & iraúna-grande \\
\hline Molothrus bonariensis (Gmelin, 1789) & vira-bosta \\
\hline Sturnella superciliaris (Bonaparte, 1850) & polícia-inglesa-do-sul \\
\hline Dolichonyx oryzivorus (Linnaeus, 1758) & triste-pia \\
\hline \multicolumn{2}{|l|}{ Fringillidae } \\
\hline Sporagra magellanica (Vieillot, 1805) & pintassilgo \\
\hline Euphonia chlorotica (Linnaeus, 1766) & fim-fim \\
\hline Euphonia violacea (Linnaeus, 1758) & gaturamo-verdadeiro \\
\hline Euphonia laniirostris d'Orbigny \& Lafresnaye, 1837 & gaturamo-de-bico-grosso \\
\hline Euphonia chalybea (Mikan, 1825) & cais-cais \\
\hline
\end{tabular}


Tab. I. Cont.

\begin{tabular}{l} 
Nome do táxon \\
\hline Euphonia cyanocephala (Vieillot, 1818) \\
Estrildidae \\
$\quad$ Estrilda astrild (Linnaeus, 1758) \\
Passeridae Rafinesque, 1815 \\
Passer domesticus (Linnaeus, 1758)
\end{tabular}

Algumas espécies poderiam ser consideradas como ocorrentes no Mato Grosso do Sul pelos registros na Reserva Biológica de Mbaracayú (PÉrez \& Colman, 1995), tal como sugerido por BORNSCHEIN (2000). No entanto, em se tratando de uma área protegida binacional cujos limites ainda não foram acordados no contexto territorial do Brasil e Paraguai, e pelo fato dos pontos específicos de contato merecerem o devido resgate, esses táxons adquiriram uma situação provisória (STRAube, 2003). Nesse sentido enquadram-se Podicephorus major (Boddaert, 1783); Gallinula melanops (Vieillot, 1819); Chaetura cinereiventris Sclater, 1862; Stephanoxis lalandi (Vieillot, 1818); Pteroglossus bailloni (Vieillot, 1819); Pygochelidon melanoleuca (Wied, 1820); Tachycineta leucopyga (Meyen, 1834); Anthus hellmayri Hartert, 1909; Agelasticus thilius (Molina, 1782) e Euphonia pectoralis (Latham, 1801).

Em virtude do ineditismo desta lista não é possível comparar riquezas nem avanços no conhecimento ornitológico do estado. No entanto, a avifauna sul-matogrossense pode ser considerada bastante diversificada, pois apesar da grande extensão territorial, essa unidade da federação não possui litoral, tampouco condições orográficas com expressivo gradiente altitudinal, tal como verificado nas porções atlânticas dos estados de São Paulo (SilveIra \& Uezu, 2011), Paraná (Scherer-Neto et al., 2011) e Rio Grande do Sul (BENCKe, 2001), que contam com 793, 744 e 624 espécies, respectivamente.

Por outro lado, o Mato Grosso do Sul abriga fitofisionomias e paisagens únicas no Brasil, tais como o Pantanal, o Chaco e os Bosques Chiquitanos. O primeiro deles corresponde a uma vasta depressão inundável do rio Paraguai, que no estado ocupa grande parte do território fronteiriço com a Bolívia e Paraguai. Nessa imensa depressão predominam as áreas abertas (campo cerrado, campos de gramíneas nativas e/ou exóticas, corixos, vazantes, baías e salinas) e, em menor proporção, os ambientes florestais, ali representados por cordilheiras (cerradão e floresta semidecídua), capões e matas de galeria ao longo do rio Paraguai e seus principais afluentes (Straube \& Pivatto, 2012).

$\mathrm{Na}$ borda oeste da planície do Pantanal o Bosque Chiquitano, um tipo especial de fitofisionomia típica da Bolívia, adentra o território brasileiro e cobre grande parte da Serra do Amolar e os morros de calcário no entorno do município de Corumbá (VASCONCElos \& HoffMAnN, 2006). Ainda na borda oeste, destaca-se o Maciço do Urucum, que exibe uma considerável variedade de tipos fitofisionômicos os quais reúnem elementos típicos de vários biomas (Cerrado, Chaco, Bosques Chiquitanos e Mata Atlântica), com predomínio de florestas estacionais semideciduais submontanas, seguidas das florestas estacionais deciduais
Nome popular

gaturamo-rei

bico-de-lacre

pardal

submontanas e, em menor representatividade, os campos das altitudes mais elevadas (Ротт et al., 2000).

A região fronteiriça com o Paraguai, no município de Porto Murtinho, a Província Chaquenha marca o limite oeste do Pantanal com presença de dois componentes básicos (ADÁMOLI, 1986): o "chaco seco" com predominância de vegetação decídua e espinhenta (diversas espécies de bromélias e cactos, bem como espécies arbóreas, notadamente aquelas do gênero Prosopis L.) e o "chaco úmido", com florestas (quebrachais Schinopsis balansae Engl. e Aspidosperma quebracho-blanco Schltdl.) e savanas (carandazais Copernicia alba Morong.) (Straube \& DI Giácomo, 2007).

Não obstante seja necessário mencionar tais situações, ressalta-se o Cerrado como o bioma mais influente na porção central e leste do estado, estendendo seus domínios até a borda leste do Pantanal (Serra de Maracaju) com predominância de fitofisionomias como cerradões (savana florestada), florestas estacionais semideciduais, vegetação ripária (mata de galeria e mata ciliar) e veredas (ADÁMOLI, 1986). A Serra de Maracaju atravessa todo o território no sentido norte-sul, atuando como um divisor de águas entre as bacias hidrográficas dos rios Paraguai (a oeste), e Paraná (a leste) (BogGiani et al., 1998).

As florestas meridionais ou atlânticas e suas transições, embora atualmente reduzidas a pequenos fragmentos, se fazem presentes na região sul de Mato Grosso do Sul e se estendem até o sudeste do estado (limite oeste da Província Atlântica), na planície alagável do alto rio Paraná (Veloso \& Strang, 1968; Gimenes et al., 2007). Na região do alto rio Paraná, a paisagem destaca-se pelos amplos canais anastomasados com reduzida declividade, ora com vasta planície inundável e grande acúmulo de sedimento em seu leito, originando pequenas ilhas, ora com ilhas grandes e planície alagável mais restrita (GIMENEs et al., 2007).

Essa notável variedade de condições propicia também a diversidade de paisagens verificada no Mato Grosso do Sul, aspecto que se revela como causa direta para a riqueza de espécies de aves listadas nesse estudo. Excetuando-se a tiriba-fogo (Pyrrhura devillei), que em território nacional ocorre somente em restrita área do Mato Grosso do Sul, de modo geral há ausência de endemismos.

As espécies aquáticas ou relacionadas a ambientes aquáticos representam $18 \%$ da comunidade de aves sulmatogrossense e estão concentradas principalmente no Pantanal e na planície de inundação do alto rio Paraná, na divisa com os estados de São Paulo e Paraná. O Pantanal abriga as maiores populações de aves aquáticas continentais ocorrentes no Brasil (NunEs \& TomAs, 2008). Vinte e sete espécies, a maioria maçaricos (Scolopacidae), inverna no Mato 
Grosso do Sul por ali passam durante seus deslocamentos do Hemisfério Norte em direção à Patagônia e vice-versa. Os visitantes meridionais (aves neotropicais) partindo da Argentina, Chile, Uruguai e extremo sul do Brasil (Rio Grande do Sul) atingem áreas mais ao norte do continente Sul Americano, como Colômbia e Venezuela (Sick, 1983).

O Estado do Mato Grosso do Sul conta com 18 espécies ameaçadas de extinção em âmbito nacional, sendo 14 inclusas na categoria de vulnerável, três em perigo e uma criticamente ameaçada. Verifica-se, no entanto, que essa condição é frágil e inconsistente, ao menos no cenário estadual, uma vez que vários táxons de distribuição restrita e/ou com sérios problemas de conservação, devido em grande parte à perda de habitat, estão fora da lista brasileira de ameaçadas de extinção (Silveira \& Straube, 2008). Listas de espécies ameaçadas são de extrema importância no planejamento de estratégias e na definição de prioridades para a conservação (GäRDENFORS, 2001). Desta forma, faz-se extremamente necessária e urgente a elaboração de uma lista de espécies ameaçadas para o Mato Grosso do Sul, através de ampla consulta e participação dos pesquisadores atuantes no estado, a exemplo do que tem sido realizado em vários outros estados brasileiros, como São Paulo (SiLVEIRA et al., 2009) e Paraná (Straube et al., 2004).

Principais grupos de pesquisa. A Ornitologia contemporânea no Estado do Mato Grosso do Sul já é uma das mais destacadas no Brasil e, embora grande parte do conhecimento até então gerado se concentre na planície do Pantanal, esforços gradativamente direcionados a diversas outras regiões do estado, caracterizam-na como emergente em pleno desenvolvimento, inclusive pelo início e desenvolvimento de grupos institucionalizados.

A unidade que mais se destaca nesse sentido é a Empresa Brasileira de Desenvolvimento Agropecuário (Embrapa-Pantanal), sediada em Corumbá para onde, graças aos inúmeros trabalhos ali desenvolvidos desde a década de 1980, converge a maior parte do conhecimento da composição estadual. Essa condição, inclusive, tem servido não somente para a produção de novas publicações sobre avifauna de localidades específicas, como para um projeto integrado e abrangente de compilação de obras instrumentais, dentre ela a Coletânea da avifauna do Estado do Mato Grosso do Sul (NunEs et al., 2007) que se encontra em fase final de preparação.

De igual importância merecem menção os estudos realizados pela Universidade Federal de Mato Grosso do Sul (UFMS), notadamente no campus de Três Lagoas, com destaque para os estudos realizados pelos ornitólogos Augusto João Piratelli, José Ragusa Netto e Sérgio Roberto Posso, cabendo a este último a responsabilidade pelo Laboratório de Ecologia, Sistemática e Conservação das Aves Neotropicais (LESCAN).

Não há como deixar de ressaltar a participação de outras universidades e demais instituições e centros de pesquisa que, embora sediados em outros estados brasileiros, se dedicam direta ou indiretamente a regiões limítrofes de seus territórios estaduais, com certa atenção nas áreas limítrofes que incluem o Mato Grosso do Sul. Graças a essas intervenções, vários setores passaram a ter sua avifauna mais conhecida, tais como a bacia do Rio Paraná (Museu de História Natural Capão da Imbuia/MHNCI, Universidade Estaduais de Londrina/UEL, Maringá/UEM e do Oeste do Paraná/UNIOESTE). Por sua importância como área úmida, o Pantanal também recebe com grande frequência a visita de estudiosos brasileiros e do exterior, por meio de projetos de organizações não governamentais empenhadas em estudos de migrações (Cemave, WWF-Brasil, Conservation International) ou de espécies ameaçadas. Em alguns casos, projetos financiados por várias entidades são sediados em pontos específicos (Fundação Neotropica, Ecotropica, projeto Arara Azul, Earthwatch, Fundação Boticário e Fundect).

$\mathrm{O}$ ecoturismo em geral e a observação de aves em particular é um elemento não somente importante como fundamental para a propulsão do conhecimento, ainda bastante preliminar, da avifauna do Mato Grosso do Sul. Situado em um dos polos turísticos mais importantes e promissores do Brasil, o Pantanal há muito tempo se destaca como área onde se concentram a maiora das visitas de observadores de aves no Mato Grosso do Sul (Straube \& Pivatto, 2012). Outra importante fonte de conhecimento sobre a avifauna do Mato Grosso do Sul são os estudos técnicos e avaliações de impactos ambientais realizados em áreas de grandes empreendimentos tais como mineradoras (e.g. Maciço do Urucum), indústrias de celulose, açúcar e álcool.

Principais acervos. Algumas instituições no exterior abrigam vários exemplares do Mato Grosso do Sul, dentre elas o Museum of Comparative Zoology (MCZ, Cambridge, EUA), o American Museum of Natural History (AMNH, Nova York, EUA), o The Field Museum of Natural History (FMNH, Chicago, EUA) e o National Museum of Natural History (NMNH, Washington, DC, EUA). No Brasil as principais coleções que guardam material ornitológico sul-matogrossense são o Museu de Zoologia (USP, São Paulo), o Museu Nacional (UFRJ, Rio de Janeiro) e o Departamento de Zoologia da Universidade Federal de Minas Gerais (DZUFMG, Minas Gerais). Há alguns exemplares depositados no Museu de História Natural Capão da Imbuia (MHNCI, Paraná), na Universidade Estadual de Londrina (UEL, Paraná), coleção da Fundação Museu de Ornitologia (FMO, Goiás) e no Laboratório de Ecologia, Sistemática e Conservação das Aves Neotropicais (UFMS, Três Lagoas). Ainda há um grande problema, extensível à literatura, que é o resgate de certas informações antigas de espécimes, em cujo campo de localidade de coleta consta apenas "Mato Grosso". Investigações futuras dos itinerários de coletores, especialmente se os exemplares estiverem acompanhados de data de coleta, poderão elucidar muitas dessas situações.

Principais lacunas de conhecimento e perspectivas de pesquisa em Ornitologia para os próximos 10 anos. Ainda existem grandes lacunas de conhecimento sobre a avifauna de Mato Grosso do Sul, notadamente em regiões limítrofes com o Paraguai (Chaco) e a Bolívia (Bosques Chiquitanos), bem como o Pantanal (sub-regiões Taquari e rio Taquari) e áreas limítrofes com o estado de Goiás. Tais 
regiões merecem atenção em futuros estudos e levantamento avifaunísticos, pois várias espécies de aves têm elevado potencial de ocorrência em território sul-matogrossense. Nesse sentido enquadram-se Micropygia schomburgkii (Schomburgk, 1848) e Sporophila minuta (Linnaeus, 1758), espécies ocorrentes no Parque Nacional de Emas (Hass, 2004), situado na região limítrofe de Goiás e Mato Grosso do Sul. No Chaco brasileiro há elevado potencial de ocorrência de Eleothreptus anomalus (Gould, 1838), Amazona vinacea (Kuhl, 1820) e Chaetura cinereiventris Sclater, 1862; ambos os táxons já foram confirmados como ocorrentes em algumas localidades no Paraguai, situadas a poucos quilômetros da fronteira com o Mato Grosso do Sul.

Agradecimentos. Agradecemos a Fundação de Apoio ao Desenvolvimento do Ensino, Ciências e Tecnologia do Estado de Mato Grosso do Sul (Fundect) e a Superintendência de Ciências e Tecnologia do Estado de Mato Grosso do Sul (Sucitec/MS) pelo convite de participação neste fascículo especial da Iheringia, Série Zoologia e o suporte financeiro para sua publicação; e a todos os pesquisadores que forneceram informações importantes sobre a avifauna de Mato Grosso do Sul: Maurício Neves Godoi, Dante R. C. Buzzetti, Sáuria L. R. Castro, Reginaldo J. Donatelli, Claudenice Faxina, Diego Hoffmann, Iêda M. Novaes Ilha, Edson Vargas Lopes, Alyson Melo, José Carlos Morante Filho, Eduardo W. Patrial, Augusto João Piratelli, Maria Antonietta C. Pivatto, José Ragusa-Netto, Luís Fábio Silveira, Walfrido M. Tomas, Alberto Urben-Filho e Marcelo Ferreira de Vasconcelos.

\section{REFERÊNCIAS BIBLIOGRÁFICAS}

AdÁmoli, J. 1986. Fitogeografia do Pantanal. In: Anais do I Simpósio sobre recursos naturais e socioeconômicos do Pantanal. Brasília, Embrapa, Ministério da Agricultura, p.105-106.

Aguirre, A. C. \& Aldrighi, A. D. 1983. Catálogo das aves do Museu da Fauna. Rio de Janeiro, IBDF. vol. I. 143p.

Aleixo, A. 2002. Molecular systematics and the role of the "varzea"-"terrafirme" ecotone in the diversification of Xiphorhynchus woodcreepers (Aves: Dendrocolaptidae). The Auk 119:621-640.

Bencke, G. A. 2001. Lista de Referência das Aves do Rio Grande do Sul. Porto Alegre, Fundação Zoobotânica do Rio Grande do Sul. 104p.

Bocchese, R. A.; Oliveira, A. K. M.; Favero, S.; Garnés, S. J. S. \& LAURA, V. A. 2008. Chuva de sementes e estabelecimento de plântulas a partir da utilização de árvores isoladas e poleiros artificiais por aves dispersoras de sementes, em área de Cerrado, Mato Grosso do Sul, Brasil. Revista Brasileira de Ornitologia 16(3):207-213.

Boggiani, P. C.; Coimbra, A. M.; Riccomini, C. \& Gesicki, A. L. D. 1998 Recursos minerais não-metálicos do Estado de Mato Grosso do Sul, Brasil. Revista do Instituto Geográfico de São Paulo 19(1/2):31-41.

Bornschein, M. R. 2000. É igualmente brasileiro o registro de Pseudocolopteryx dinellianus (Passeriformes: Tyrannidae) para o Refúgio Biológico de Maracaju, uma reserva binacional (ParaguaiBrasil). Nattereria 1:23-24.

Carlos, C. J.; Straube, F. C. \& Pacheco, J. F. 2010. Conceitos e definições sobre documentação de registros ornitológicos e critérios para a elaboração de listas de aves para os estados brasileiros. Revista Brasileira de Ornitologia 18(4):355-361.

CBRO - Comitê Brasileiro de Registros Ornitológicos. 2009. Listas das aves do Brasil. 8ed - 09.08.2009. Disponível em: $<$ http://www.cbro. org.br/CBRO/pdf/avesbrasil_ago2009.pdf > . Acessado em: 14.03.2012.

CBRO - Comitê Brasileiro de Registros Ornitológicos. 2011. Listas das aves do Brasil. 10ed. - 25.01.2011. Disponível em: $<\mathrm{http}: / /$ www.cbro.org.br/CBRO/pdf/avesbrasil_jan2011.pdf $>$. Acessado em 14.03.2012.

Collar, N. J.; Gonzaga, L. P.; Krabbe, N.; Madroño Nieto, A.; Naranjo, L. G.; PARKER III, T. A. \& Wege, D. 1992. Threatened birds of Americas. The ICBP/IUCN red data book. Cambridge, International Council for Bird Preservation. 1150p.
D’Horta, F. M.; Silva, J. M. C. \& Ribas, C. C. 2008. Species limits and hybridization zones in Icterus cayanensis-chrysocephalus group (Aves: Icteridae). Biological Journal of the Linnean Society 95:583-597.

Donatelli, R. 2005. Birds and dynamics habitat mosaics in the Pantanal. In: Chandler, M.; Wang, E. \& Johansson, E. P. eds. The Pantanal Conservation Research Initiative. Boston, Earthwatch Institute, p. 50-69.

Endrigo, E. R. 2005. Bird List Pousada Aguapé. Disponível em: <http:// www.aguape.com.br/lista.swf $>$. Acessado em 29.10.2010.

Farias, G. B. \& Alves, A. G. C. 2007. É importante pesquisar o nome local das aves? Revista Brasileira de Ornitologia 15(3):403-408.

GärdENFORs, U. 2001. Classifying threatened species at national versus global levels. Trends in Ecology and Evolution 16:511-516.

Gimenes, M. R.; Lopes, E. V.; Loures-Ribeiro, A.; MendonçA, L. B. \& Anjos, L. 2007. Aves da planície alagável do alto rio Paraná. Maringá, Editora da Universidade Estadual de Maringá. 281p.

Grau, E. T.; Pereira, S. L.; Silveira, L. F.; HöFling, E. \& Wajntal, A. 2004. Molecular phylogenetics and biogeography of Neotropical piping guans (Aves: Galliformes): Pipile Bonaparte, 1856 is synonym of Aburria Reichenbach, 1853. Molecular Phylogenetics and Evolution 35:637-645.

Hass, A. 2004. Aves. In: Plano de Manejo do Parque Nacional das Emas (IBAMA). Brasília, Ministério do Meio Ambiente, p. 2.19-2.25.

Hughes, B.; Dugger, B.; Cunha, H. J.; lamas, I. R.; GoERCK, J. M.; Lins, L. V.; Silveira, L. F.; Andrade, R. D. D.; Bruno, S. F.; Rigueira, S. E. \& Barros, Y. D. M. 2006. Plano de ação para a conservação do pato-mergulhão Mergus octosetaceus. Brasília, IBAMA. 8p.

IherING, H. von. 1898. As aves do Estado de São Paulo. Revista do Museu Paulista 3:113-476.

IHERING, H. von. 1899. As aves do Estado do Rio Grande do Sul. In: Anuário do Estado do Rio Grande do Sul para o ano de 1900. Porto Alegre, Governo do Estado do Rio Grande do Sul, p.113-154.

Ihering, H. von. 1900. Aves de S. Paulo. Revista do Museu Paulista 4:1-20.

IhERING, H. vON. 1904. As aves do Paraguay em comparação com as de São Paulo. Revista do Museu Paulista 6:310-384.

Leuzinger, L. 2009. Fazenda Barranco Alto - Birdlist. Disponível em: <http://www.pantanalbirds.com/Birdlist_Pantanal_FBAL.pdf $>$. Acessado em 28.10.2010.

LOPES, P. 2009. Taxonomia alfa e distribuição dos representantes do gênero Aburria Reichenbach, 1853 (Aves: Cracidae). Dissertação de Mestrado. São Paulo, Universidade de São Paulo. 158p.

Melo, F. 2006. Refúgio Ecológico Caiman (Pantanal, Brasil): listas de fauna e flora. Miranda, Pousada Caiman. Disponível em: $<\mathrm{http}: /$ www.caiman.com.br/caiman/new-portugues/pantanal/fauna.asp>. Acessado em 28.10.2010.

Morrison, R. G.; Serrano, I. D. L.; Antas, P. D. T. Z. \& Ross, R. K. 2008. Aves migratórias no Pantanal. Distribuição de aves limícolas neárticas e outras espécies aquáticas no Pantanal. Brasília, WWFBrasil, 100p.

NACINOVIC, J. B. 2012. Uma contribuição iconográfica para a identificação de jacus (Aves, Cracidae, Penelope) que ocorrem no Brasil. Rio de Janeiro, Agência $2^{\circ}$. Comunicação.

NaumburG, E. M. B. 1930. The Birds of Mato Grosso, Brazil: a report on the birds secured by the Roosevelt-Rondon expedition. Bulletin of the American Museum of Natural History 60:1-431.

Nunes, A. P.; SilvV, P. A. \& TomAs, W. M. 2008. Novos registros de aves para o Pantanal, Brasil. Revista Brasileira de Ornitologia 16(2):160-164.

Nunes, A. P.; Tizianel, F. A. T.; Melo, A. V.; Nascimento, V. \& Machado, N. 2010. Aves da Estrada Parque Pantanal, Corumbá, Mato Grosso do Sul, Brasil. Atualidades Ornitológicas 156:33-47.

Nunes, A. P.; Tizianeli, F. A. T.; Tomas, W. M. \& Lupinetti, C. 2009. Aves da fazenda Nhumirim e seus arredores: Lista 2008. Boletim de Pesquisa e Desenvolvimento, EMBRAPA-CPAP 89:1-44.

Nunes, A. P. \& Tomas, W. M. 2008. Aves migratórias e nômades ocorrentes no Pantanal. Corumbá, Embrapa-CPAP. 124p.

Nunes, A. P.; Straube, F. C.; LaPs, R. R. \& Posso, S. R. 2017. Checklist das aves do Estado do Mato Grosso do Sul, Brasil. Iheringia, Série Zoologia 107(supl.):e2017154.

OMLAND, K. E.; LANYON, S. M. \& FrITZ, S. J. 1999. A molecular phylogeny of the New World orioles (Icterus): the importance of dense taxon sampling. Molecular Phylogenetics and Evolution 12:224-239. 
Pacheco, J. F. 2004. Ocorrência acidental de Buteo polyosoma (Quoy \& Gaimard, 1824) na Ilha de Cabo Frio, Rio de Janeiro, Brasil. Ararajuba 12:168-169.

Pacheco, J. F. \& Bauer, C. 2001. A lista de aves do Espírito Santo de Ruschi (1953): uma análise crítica. In: Albuquerque, J. L. B.; CANDido Junior, J. F.; Straube, F. C. \& Roos, A. L. eds. Ornitologia e conservação: da ciência às estratégias. Tubarão, Unisul. p.261-276.

PARKER, T. A. \& Willis, E. O. 1997. Notes on three tiny grassland flycatchers, with comments on the disappearance of South American fire-diversified savannas. Ornithological Monographs 48:549-555.

Pérez, V. \& Colman, J. 1995. Avifauna de las areas protegidas de Itaipu. I. Aves del Refugio Biologico Mbaracayu, Salto de Guayra Paraguay. Biota 4:1-4.

Pinto, O. M. O. 1952. Súmula histórica e sistemática da ornitologia de Minas Gerais. Arquivos de Zoologia do Estado de São Paulo 8(1):1-51.

Pinto, O. M. O. 1978. Novo catálogo das aves do Brasil. Aves não Passeriformes e Passeriformes não Oscines, com exclusão da família Tyrannidae. São Paulo, Empresa Gráfica Revista dos Tribunais. vol.1. $446 \mathrm{p}$.

Pivatto, M. A. C.; Donatelli, R. J. \& Manço, D. D. M. 2008. Aves da fazenda Santa Emília, Aquidauana, Mato Grosso do Sul. Atualidades Ornitológicas 143:33-37.

Posso, S. R.; Bueno, F. A.; Previatto, D. M.; Pinheiro, L. C.; Mizobe, R. S.; Berni, M. A.; Taloni, F. D. \& Cintra, F. 2008. Levantamento da avifauna e avaliação ambiental do Parque das Capivaras, Três Lagoas, MS. In: Anais do XXVII Congresso Brasileiro de Zoologia. Curitiba, Sociedade Brasileira de Zoologia (cd Room).

Posso, S. R.; Bueno, F. A.; Mizobe, R. S.; Pinheiro, L. C.; Previatto, D. M.; Dias, V. B.; Filgueiras, V.; Lima, B. M.; Cancian, D.; Miyaji, E. E. \& Morante Filho, J. C. 2009. Levantamento preliminar da avifauna da Reserva Cisalpina - Brasilândia, Mato Grosso do Sul. In: XVII Congresso Brasileiro de Ornitologia, Anais do XVII Congresso Brasileiro de Ornitologia. Aracruz, Sociedade Brasileira de Ornitologia.

Pott, A.; Silva, J. S. V.; SAlis, S. M.; Pott, V. J. \& Silva, M. P. 2000. Vegetação e uso da terra. In: SILVA, J. S. V. org. Zoneamento ambiental da borda oeste do Pantanal: Maciço do Urucum e adjacências. Brasília, Embrapa, Comunicação e Transferência de Tecnologia, p.111-131.

Previatto, D. M.; Mizobe, R. S.; Bueno, F. A.; Pinheiro, L. C. \& Posso, S. R. 2009. Exploração dos recursos florais de Spathodea nilotica (Bignoniaceae) por aves na área urbana de Três Lagoas/MS. In: XVII Congresso Brasileiro de Ornitologia, Anais do XVII Congresso Brasileiro de Ornitologia. Aracruz, Sociedade Brasileira de Ornitologia.

Ragusa-NetTo, J. 2002. Fruting phenology and consumption by birds in Ficus calyptroceras (Miq.) Miq. (Moraceae). Brazilian Journal of Biology 62(2):339-346.

ReichHolf, J. H. 1974. Artenreichtum, Häufigkeit und Diversität der Greifvögel in einigen Gebieten von Südamerika. Journal of Ornithology 115:381-397.

Ridgely, R. S. \& Tudor, G. 1994. The Birds of South America. The Suboscines Passerines. Austin, University of Texas Press. 940p.

Ruschi, A. 1953. Lista das aves do Estado do Espírito Santo. Boletim do Museu de Biologia Mello Leitão, Série Zoologia 11:1-21.

Scherer-Neto, P.; Straube, F. C.; Carrano, E. \& Urben-Filho, A. 2011. Lista das aves do Paraná. Edição comemorativa do "Centenário da Ornitologia do Paraná". Curitiba, Hori Consultoria Ambiental. 130p.

Schubart, O.; Aguirre, A. C. \& Sick, H. 1965. Contribuição para o conhecimento da alimentação das aves brasileiras. Arquivos de Zoologia do Estado de São Paulo 12:95-249.

SHORT, L. L., JR. 1975. A zoogeographical analysis of the South American Chaco avifauna. Bulletin of the American Museum of Natural History 154:163-352.

SICK, H. 1983. Migrações de aves na América do Sul continental. Brasília, Instituto Brasileiro de Desenvolvimento Florestal. 86p.
SICK, H. 1985. Ornitologia brasileira: uma introdução. Brasília, Editora UnB. 827 p.

Sick, H. 1997. Ornitologia brasileira. Rio de Janeiro, Nova Fronteira. 912p.

Sick, H. \& TeIXeIRA, D. M. 1979. Notas sobre aves brasileiras raras ou ameaçadas de extinção. Publicações Avulsas do Museu Nacional 62:1-39.

Silva, J. M. C. 1995. Birds of the Cerrado region, South America. Steenstrupia 21:69-92.

Silva, M. B.; Zucca, C. F.; Souza, C. R.; Mamede, S.; Pina, P. I. \& Oliveira, I. R. 2006. Inventário da avifauna no complexo AporéSucuriú. In: Pagotto, T. C. S. \& SouzA, P. R. eds. Biodiversidade do complexo Aporé-Sucuriú: subsídios à conservação e manejo do bioma Cerrado. Campo Grande, Editora da Universidade Federal de Mato Grosso do Sul, p.116-128.

Silveira, L. F.; Benedicto, G. A.; Schunck, F. \& Sugieda, A. M. 2009. Aves. In: Bressan, P. M.; Kierulff, M. C. M. \& Sugieda, A. M. coord. Fauna ameaçada de extinção no Estado de São Paulo. Vertebrados. São Paulo, Governo do Estado de Minas Gerais/Secretaria do Meio Ambiente, Fundação Parque Zoológico de São Paulo, p. 87-283.

Silveira, L. F.; Beisiegel, B. M.; Curcio, F. F.; Valdujo, P. H.; Dixo, M.; Verdade, V. K.; Mattox, G. M. T. \& Cunningham, P. T. M. 2010. Para que servem os inventários de fauna? Estudos Avançados 24(68):173-207.

Silveira, L. F. \& Straube, F. C. 2008. Aves ameaçadas de extinção no Brasil. In: Machado, A. B. M.; Drummond, G. M. \& Paglia, A. P. eds. Livro Vermelho da Fauna Brasileira Ameaçada de Extinção. Brasília, Ministério do Meio Ambiente/Fundação Biodiversitas, p.379-666.

Silveira, L. F. \& UezU, A. 2011. Checklist das aves do Estado de São Paulo, Brasil. Biota Neotropica 11(1a):1-28. Disponível em: <http://www. biotaneotropica.org.br/v11n1a/pt/abstract?inventory+bn0061101a201>.

SouzA, D. 2005. Um exemplar esquecido do jacupixuna Penelope obscura, oriundo de Mato Grosso do Sul. Atualidades Ornitológicas 128:11.

Straube, F. C. 2003. Bases legais para a identificação dos limites territoriais do Brasil na fronteira com o Paraguai e suas implicações para a consideração de registros ornitológicos. Ararajuba 11(1):131-145.

Straube, F. C. \& Di Giácomo, A. 2007. Avifauna das regiões subtropical e temperada do Neotrópico: desafios biogeográficos. Ciências do Ambiente 35:137-166.

Straube, F. C. \& Pivatto, M. A. C. 2012. O Pantanal do Mato Grosso do Sul: destino para a observação de aves. Atualidades Ornitológicas 167:33-50.

Straube, F. C. \& Scherer-Neto, P. 1995 Novas observações sobre o "cunhataí" Triclaria malachitacea (Spix, 1824) nos Estados de Paraná e São Paulo. Acta Biologica Leopoldina 17(1):147-152.

Straube, F. C.; Urben-Filho, A. \& Kajiwara, D. 2004. Aves. In: Mikich, S. B. \& BÉRnILS, B. S. eds. Livro vermelho da fauna ameaçada no estado do Paraná. Curitiba, Instituto Ambiental do Paraná, p.145-496.

Straube, F. C.; Urben-Filho, A.; Pivatto, M. A. C.; Nunes, A. P. \& Tomas, W. M. 2006. Nova contribuição à ornitologia do Chaco Brasileiro (Mato Grosso do Sul, Brasil). Atualidades Ornitológicas 134:1-27.

Sturge, R.; Jacobsen, F.; Rosensteel, B. B.; Neale, R. J. \& Omland, K. E. 2009. Colonization of South America from Caribbean Islands confirmed by molecular phylogeny with increased taxon sampling. Condor 11:575-579

Travassos, L. 1940. Relatório da terceira excursão a zona da Estrada de Ferro Noroeste do Brasil realizada em fevereiro e março de 1940. Memórias do Instituto Oswaldo Cruz 35(3):607-696.

Tubelis, D. P. \& Tomas, W. M. 2003. Bird species of the wetland, Brazil. Ararajuba 11(1):5-37.

Vasconcelos, M. F. \& Hoffmann, D. 2006. Os Bosques Secos Chiquitanos também são nossos! Atualidades Ornitológicas 130:10-11.

Veloso, H. P. \& Strang, H. E. 1968. Aspectos da fitofisionomia do sul do Estado de Mato Grosso. Memórias do Instituto Oswaldo Cruz 68(1):77-88. 
Apêndice 1. Lista terciária das aves do estado do Mato Grosso do Sul, Brasil.

\begin{tabular}{lc}
\hline Táxon & Nome popular \\
Fonte de citação e racionália & codorna-do-nordeste \\
\hline Tinamidae & Nothura boraquira (Spix, 1825) \\
STRAuBE et al. (2006): embora a espécie tenha sido tentativamente identificada com base na literatura e comparação com exemplares de museu, a \\
identificação é frágil, pela falta de documentação e da semelhança conhecida entre outras espécies, inclusive Nothoprocta cinerascens (Burmeister, \\
1860) que, embora ainda não registrada no País, ocorre marginalmente ao Mato Grosso do Sul, precisamente na região onde ocorreu o contato \\
(SHORT, 1975).
\end{tabular}
Anatidae
Anas sibilatrix Poeppig, 1829
marreca-oveira

Melo (2006): menção em lista de espécies, sem circunstanciação; a distribuição é incompatível.

Anas cyanoptera Vieillot, 1816 marreca-colorada

Nunes et al. (2008): os dados alusivos ao registro não puderam ser reavaliados.

Mergus octosetaceus Vieillot, 1817 pato-mergulhão

Collar et al. (1992): menção para bacia do Sucuriú, sem circunstanciação; Hughes et al. (2006) descartam esse registro.

Cracidae

Penelope obscura Temminck, $1815 \quad$ jacuaçu

Aguirre \& Aldrighi (1983), Souza (2005): o exemplar atribuído à espécie foi analisado por NACINOviC (2012) que procedeu a correção da identificação para P. ochrogaster.

Accipitridae

Geranoaetus polyosoma (Quoy \& Gaimard, 1824) gavião-de-costas-vermelhas

ReichHolf (1974), PACHeco (2004): menção em lista de espécies, sem circunstanciação, localidades ou datas.

Rallidae

Fulica leucoptera Vieillot, $1817 \quad$ carqueja-de-bico-amarelo

LEUZINGER (2009): menção em lista de espécies, sem detalhamento; a distribuição é incompatível.

Scolopacidae

Numenius phaeopus (Linnaeus, 1758) maçarico-galego

MORRISON et al. (2008): menção em obra de referência, sem circunstanciação.

Arenaria interpres (Linnaeus, 1758) vira-pedras

MorRISON et al. (2008): menção em obra de referência, sem circunstanciação.

Calidris alba (Pallas, 1764) maçarico-branco

Tubelis \& Tomas (2003): menção em lista de espécies. Equívoco de identificação. De acordo com STRAUBE et al. (2006), os espécimes procedentes de Porto Quebracho e identificados no Museu Nacional como tal, são Phalaropus tricolor (Vieillot, 1819).

Calidris pusilla (Linnaeus, 1766) maçarico-rasteirinho

TuBelis \& Tomas (2003): registro único, não documentado do segundo autor, que não pôde ser reavaliado.

Calidris minutilla (Vieillot, 1819)

LEUZINGER (2009): citação em lista de espécies, sem circunstanciação.

Calidris bairdii (Coues, 1861) maçarico-de-bico-fino

Melo (2006): menção em lista de espécies, sem circunstanciação; conforme STRAubE et al. (2006), os espécimes identificados no Museu Nacional como tal, se tratam de Calidris melanotos (Vieillot, 1819); a espécie é de difícil identificação e geralmente distribuída pelo litoral meridional do Brasil.

Columbidae

Columbina cyanopis (Pelzeln, 1870) rolinha-do-planalto

PARKER \& WiLlis (1997): registro único, não documentado e carente de confirmação.

Psittacidae

Anodorhynchus glaucus (Vieillot, 1816) arara-azul-pequena

Collar et al. (1992): informação sem detalhamento geográfica, baseada em especulações.

Pionopsitta pileata (Scopoli, 1769) cuiú-cuiú

BocCHeSE et al. (2008): citação em lista de espécies, sem circunstanciação. Distribuição incompatível.

Triclaria malachitacea (Spix, 1824) sabiá-cica

Sick \& TeIXeIra (1979), Sick (1985): a indicação para “Campo Grande”, foi satisfatoriamente refutada por STRAUBE \& Scherer-Neto (1995), situação acatada em SICK (1997).

Strigidae

Aegolius harrisii (Cassin, 1849)

Hass (2004): menção em lista de espécies, sem circunstanciação.

Caprimulgidae

Hydropsalis forcipata (Nitzsch, 1840) bacurau-tesoura-gigante

ENDRIGO (2005): menção em lista de espécies, sem circunstanciação; o autor (Endrigo, 2012 in litt.) confirmou o equívoco.

Apodidae

Chaetura brachyura (Jardine, 1846)

andorinhão-de-rabo-curto

Travassos (1940), Naumburg (1930): erro de identificação: o exemplar coletado em Salobra e depositado no Museu Nacional corresponde ao congênere $C$. meridionalis e o exemplar no American Museum of Natural History carece de confirmação quanto à identidade.

Trochilidae

Phaethornis ruber (Linnaeus, 1758)

rabo-branco-rubro

Hass (2004): menção em lista de espécies, sem circunstanciação. Distribuição incompatível.

Phaethornis eurynome (Lesson, 1832)

rabo-branco-de-garganta-rajada

Tubelis \& Tomas (2003), Nunes et al. (2009): menção em listas de espécies, sem ciircunstanciação. 
Apêndice 1. Cont.

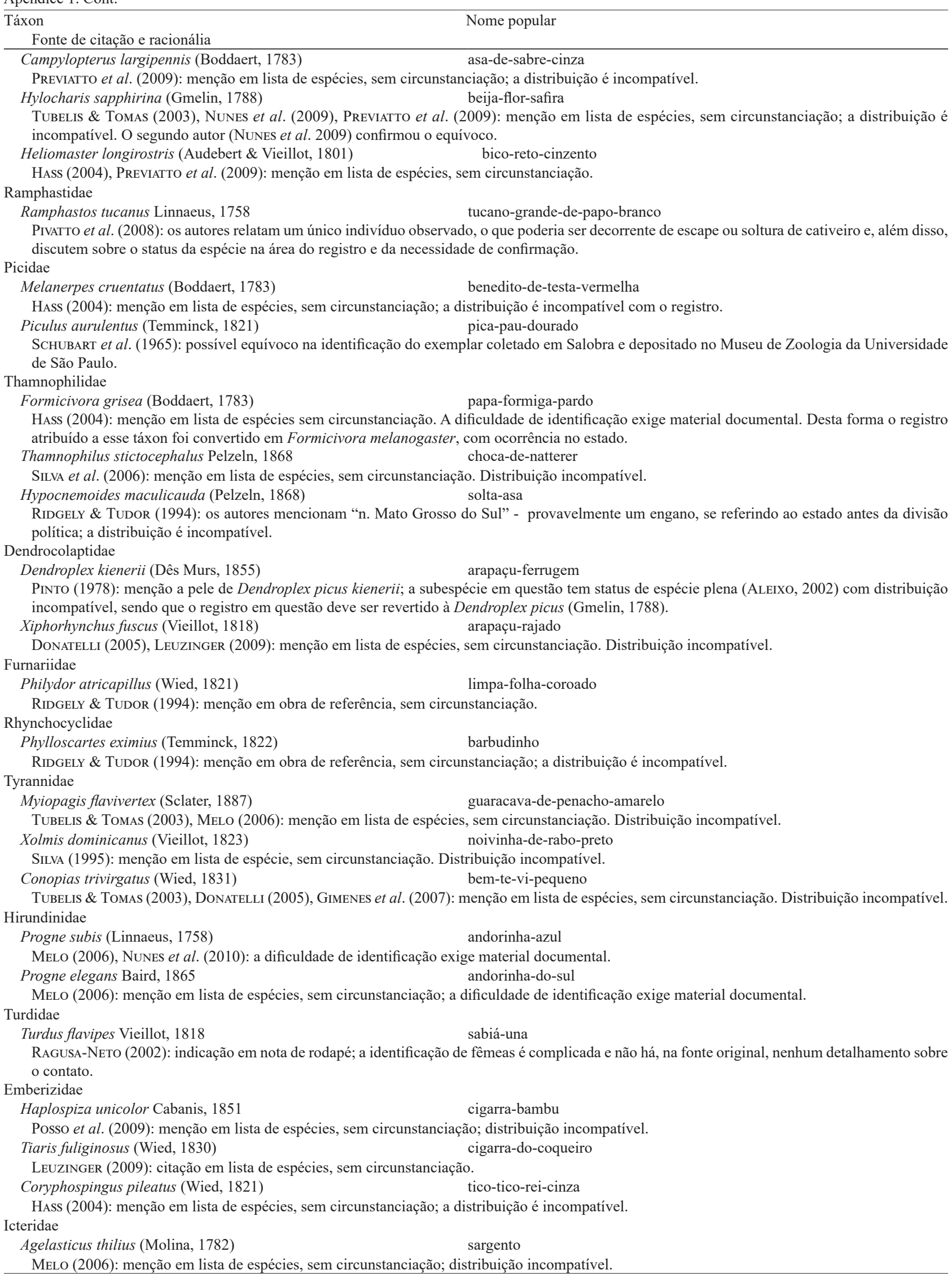

